\title{
ON THE FLORA OF OUTCH.
}

BY

E. BLATTER, s.J.

PAlt I.

The flora of Cutch has receiven very liftle attention on the part of botanical explorers. There is scrarcely any other purt in India that is so seldom mentioned in lotistic works as Cntel. Mueh less are we able to find any special publieation on its vagetation. When Hooker and Thomson publisbed their "Flora Indica" in 1555, they gave us in the "Introluctory Essay" at valuable accomnt of the physical and botanical features of the various prart of India, but regarding Cutch we read only the following remarks : "The district of Kach, wheh is separateil from Katiwar loy the Gulf of Kach, a narrow arm of the sea, from Sindli by the most eastem bamch of the Indus, and from Marwar by the Ranu (a very singular saline and more or less marshy phain in which the river Lmi loses itoelf) has a very similar clinate to the peninsula of Gujarat, being like that iraversed by a range of hills running liom Whest to Eust. It may, therefore, (for our purposes) with more propriety he considered at part of Gujarat, than to betong to Sindh, to which physically an well as politically it is more nearly related. The northern listricts of loth Kach and Katiwar, being ereened from the min-bringing winds by the bills, are extremely arid," 1 Since the time when this was written, the countries smrounding Cutch have been esplored more miuntely as regards their filora, and the physical and meteorologieal condition prevailing in them are heiter known. This will enable us later on to decile the question whether Cuteh ought to be considered a part of the botanical frovince of Gujarat or of Sind. The last 50 years did not add mueh to our knowledge of the Alori of Cutch. Here we are peaking of the printed records only, for wo are not so fortunate as to have aceess to the extensive herbaria of Europe which very likely contain specimens also of Cutch. Of the former only one came under our notice. It is a list of the plants of Cuteh, prepared by Colonel U. T. Palin as a contribution to Vol. V. of the Bombay Gazetteer in i 880 . The circumstance 'tat the habitat and flowering time is added to the names of many species make the catalogue a very valuable

1. Houker, J, D., and Thomson, T., Flora Indica, Vo!. I., p. 150. 


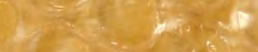

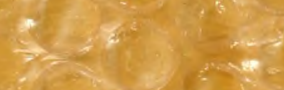

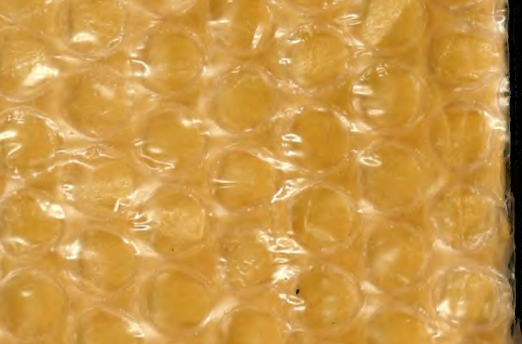

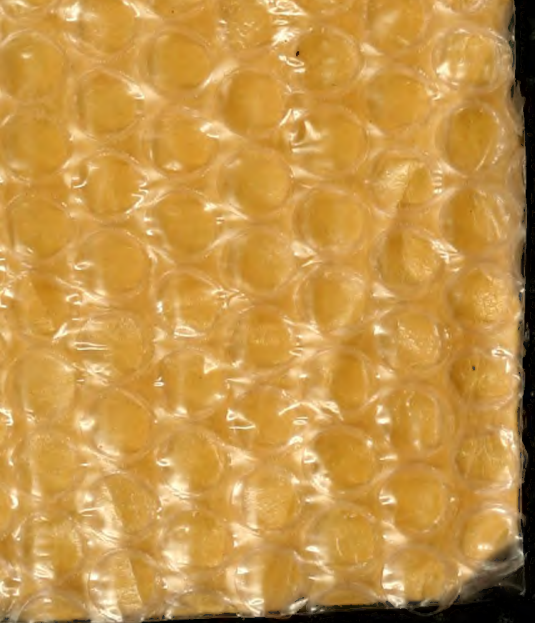

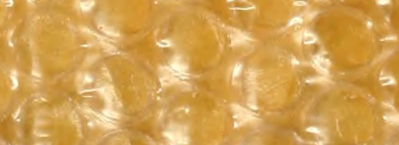


ON THE FLORA OF CUTCH.

BY

E. BuAtтer, s...

Part I.

The flora of Cutch has receivel rery little attention on the part of I) stanical explorers. There is sareely any other part in India that is so seldom mentioned in loristic works as Cnteh. Much less a re we able to find any special publication on its vagetation. When Hooker and Thomson prolished their "Flen Indica" in 18.5, they gave ns in the "Introlnctory Essay" at valuable account of the physical and botanical features of the varions part of India, but regarding Cutah we read only the following remarks: "The district of Kach, which in separated from Katiwar by the Gulf of Kach, a narrow arm of the sea, from Sindh by the most eastern banch of the Indus, and from Marwar by the Ramn (a very simgular saliw and more or less marshy plain in which the river Lmini loses itolf) has a very similar climate to the peninsula of Gujarat, being like that iravered by a range of hills rumning firom West to East. It may, therefore, (for our purposes) with more propriety he considered a part of Gujarat, than to belong to sindh, to which physicully an well ats politically it is more nearly related. The nerthem districts of both Kash and Katiwar, being serened from the rain-bringing wind by the bills, are extremely arid." 1 Since the time when this was written, the ovuntries surounding Cutch bave been explored more mimutely as regards their dion, and the physigal and meteorologieal condition prevailing in them are beiter known. This will enable us later on to decide the question whether Cutch ought to be considered a part of the botanical province of Gujarat or of Sind. The last 50 years did not add much to our knowledge of the dora of Cutch. Here we are peaking of the printed records only, for we are not so fortunate as to have access to the extensive herbaria of Europe which very likely rontain specimens also of Cutch. Of the former only one came under cour notice. It is a list of the plants of Cutch, prepared by Colonel C. T. Palin as a contribution to Vol. V. of the Bombay Gazetteer in i880. The cireumstance "Jat the habitat and flowering time is added to the names of many species make the eatalogue a very valuable

1. Houker, J, D., and Thomson, T, Flora Indica, Vo!. I., p. 150. 
349.5
B. 58 
one. It was just the fact, on the one lind, that the flora of Cutch is very ineompletely known and, on the other, that Gutch occupies an almost isolated po-ition, that mulued ne in December last, io pay is vi-it to that out-ot-the-way comery. If 1 am now able to auld something to our previous knowledge of the Botumy of Cnreh, it is greatly whe to the untiring efforts of my companiom, the Res. H. Sierp, s.t., l'rotessor of Chemistry, who, with valuable slaggestions and practical ahi, wa- at great help to me thronghont the whole journey.

As the physical aspeth and meteorological com litions of a country detemine the special clameter and development of it regetation, it is advisable to give first a short sketch of the territory of Cutch. I comsider this less superifuns because compantively very few are personally acquanterl with Cutch, and if others try to form an idea as to its physieal configuration, I an afraid, the pieture they draw of C'ntch will not be accurate, not to say incorrect, if I am allowed to draw a conclusion from my own experiences.

The Propince of Cutch extends from $20^{\circ} 4 \tau^{\prime}$ to $24^{\circ} \mathrm{N}$. Lat, and $68026^{\prime}$ to $71^{\circ} 10^{\prime} \mathrm{E}$. Long., being crossed by the parallel of the tropic of eancer about 14 miles north of the eapital, Bhuj. On the nortl, east, and south-east it is bounded by the Ramin; on the south by the Gulf of Cutch, on the west by the Arabian Sea, and on the northwest by the eastem branch of the Indus. Its extreme length from east to west is 160 miles an? its extreme breath 70 from north to south, whike in one place it is only 35 miles wide. It contains about 6,500 square mile: exclusive of the Grand Ramm, which, incluting the islands with the portion bounded by the Gujarat Coast in the east and suththast corers an area of 10,000 square miles. Perlaps the most striking feature to the visitor of Gutch is the sterility of the country. Sandy plains and naked rocky hils present a strong contrast to the more fertile parts of India. The barremens is increased by the seareity of trees and general alusence of anything that can be called jungle. "From the sea on the sonth and west, and from the Ramn on the north and east, the coast of ('utch is in some places rery slightly raised and fringed with mangrove swanps. In other parts it risein rows of sand hills, or as in the north-west, in broken rocky eliffs. Inland, especially on the soutn and east, are hroad plains, some deep soiled and well tilled, others bare and furrowed with water-courses. Beyond these plains rive the central lands of the province, in places 

relieved by bright coloured rocks and patches of tillage, but over most of the area brown waving uplands deep in loose sand, broken by naked peaks, and bordere 1 by lare ridges of low dust-coloured hilis." 1 Yet parts of the country are far from being unpicturesque. Though none of the elexations of Cutch are very great, being generally under 1,500 feet above the sea, its hills form one of the chief natural featres of the Province. Three distinct ranges of hills are iraceable in Cutch proper having an easterly and westerly direetion. The most northem range overlooks the Rann from near Luliput to a point about 28 miles eastward of Bhuj. It forms an irregular chain, and, for the greater part, presents to the north a perpendicular cliff, and to the south an inclined plain. The next called the Charwar range, passes transversely through the centre of the Province, and is connected with the former, at its north-western extremity, by a cluster of hills. It is made up. partly of sundstone. and partly of a series of strata of slate clay, limestone, slate, and slaty limestone. The third, or southern range called the Dora hils, has the same general direction as the other two, but is of -smaller extent and cousisis entirely of volcanic materials. A number of isolated volcanic hills are also seutlered over the plain, as well as in other parts of the Province, rarticularly on the borders of the Liam, where is situated the hill Dhinodhar, the highest in Cutch (1 073 feet). The Fagad hills in the eatern part of the district are a broad group stretching east und west. They have many separate peaks, the principal of which is the Vittroe. A disconnected chain traverses the Rimn islands of Pachan, Kurreer, Bela. and Chorar from west to east. The inost lofty summit oecurs at Pachan Peer, rising 1437 feot alove the liann. The variel and vivid eolouring of the rocks adel a peculiar charm to the aspect of the cotantry. The three primary colours with many varieties of their compounds are represented by the rocks us well as their atmospheric debris. "Owing to the oxides of iron, red and yellow prevuil: but in some places these are mixed with pale lavender, blue and purple tints, and contrasied with intense black or the purest white; and when any vegetation adds some green, the brilliancy of the effect becomes very striking. As a rule, the country has warn sienna tints, with red, purple or black rocks nearly always close at band; while the grey, purple or orange hills 

are often varied by patehes of white strongly relieved against the arijacent sombre colour of some mass of intrasive or overlying trup."

There are no rivers that he vater enough to flow throughout the vear. The river conrses are merely channels for conveying the periolical thoods from the central uplands to the sea and Rann respectively. The Kliari, which rises in the Chorad hills, about eight milus south-west of Bhuj, has a course of about 30 miles. Flowing past Bhuj and winding its way between steep banks in places 110 feet hygh, it keeps north and loses itself in the Rann. The largest rivers that have a southern direction are the Madh and the 'T'erab. They flow for about 30 miles across the Abdasi plain and fall together mto the Gulf of Cutch. Owing to the fict that amost all the rocks are impregnatrit with salts, the water of the Cuteh streams is infit to drink, and during the hot season is too salt even for cattle. This circunstance has to dh it great deal with the character of the present flora. Water is nstally. found at no great depth from the surfiee. Many wells being 15 th t5 feet deep yield snfficient supplies. The pousds, which are not nucominon, are mostly small and usually sun dry in sis months.?

As to the elimate of Cutch we have to rely almost entirely un general and insufficient data scatterel here and there in variondescriptions of that Province ${ }^{3}$. Being situated along the north pamilel of the tropic of Cancer, Gutch is very little subjected to the rainbringing influence of the sonth-west monson. Though heary monson rains are experienced on the western shores and side of India far to the north of Ahmedabad, they seem to neglect the sonthern parts of Sind and Kattiawas. The 21 years preceding 186,

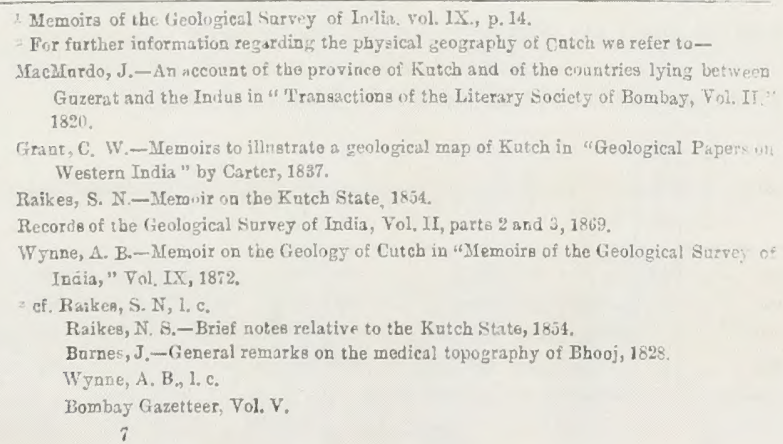



Aher an average ammul rabiall of $11 \cdot 30$ inches at Bhuj, the max-

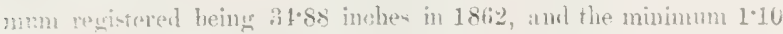
in 1818. The min generally reache- futeh from directinn oppusiu te ilat of the prevailing winds, in the form of wpalls from the noth-

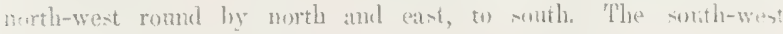

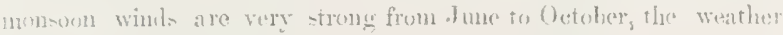
heing seletom calm. In the cold monthe harth eats and north winds

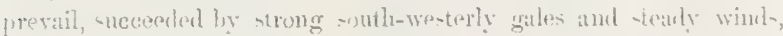

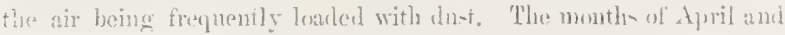
Miay are very hot inlaml, while along the eomat the climate is delizhtful, from the alwence of the huming winds and dut atome that prevail elsewhere during thow monthe In June the lost wind

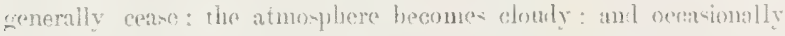
the mins, which ammenes rery irregularly, are precenterl by

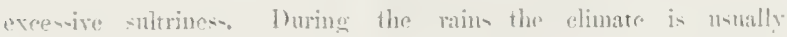
mo-t agreeahle: clomly and exol, with a eool breeze night and day. The cold wather sots in later in ('uteh than in Gujarat. The - mu during the first half of Xorember in oppresively loot: Deember is

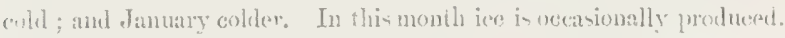
In Marth, again, the smu hegins to strike hot.

Through the kinduess of Dr. P. .J. Fignterede, whe is in charege of

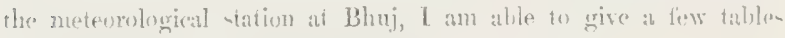

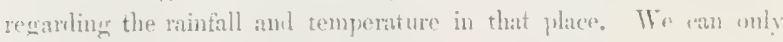
renret that similar obserations are mot made in other places of the province, as it is very probalile that distinct differenees exist in the flain- nerth and south of the hill ranges. The differences will, how"ver, not be very great, and the snbjoined data are likely in girs a finiv ilen of the climate of the whole of Cutch.

lihnj IRaintall, 1904-1907, at N. Lat. $230155^{\prime}$, and L. Long. 6904!'.

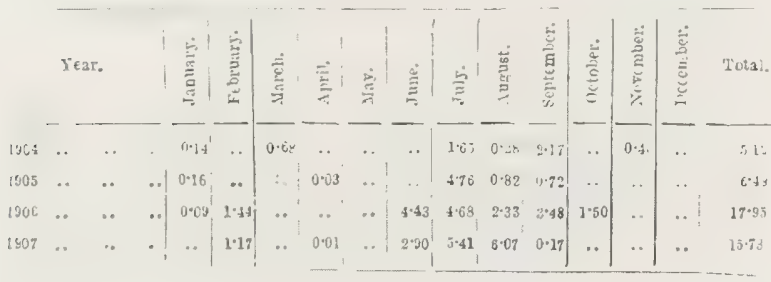





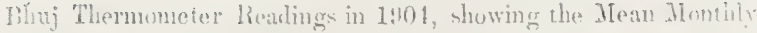
Maxima and Minima with Mean Maily Rimce.

\begin{tabular}{|c|c|c|c|c|c|c|c|c|c|c|c|c|c|c|c|c|c|}
\hline \multicolumn{3}{|c|}{ Januars. } & \multicolumn{3}{|c|}{ Feortu.sts. } & \multicolumn{3}{|c|}{ Mnrch, } & \multicolumn{3}{|c|}{ Aprll. } & \multicolumn{3}{|c|}{ Mas. } & \multicolumn{3}{|c|}{ June. } \\
\hline 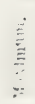 & 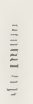 & 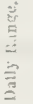 & ह & $\frac{\dot{a}}{\stackrel{a}{E}}$ & 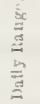 & $\stackrel{\vec{E}}{\stackrel{E}{E}}$ & 䔍 & 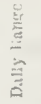 & $\frac{B}{2}$ & 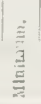 & 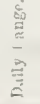 & 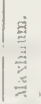 & 离 & 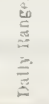 & $\begin{array}{l}\text { E } \\
\text { E } \\
\text { E } \\
\text { E }\end{array}$ & $\vec{E}$ & $\begin{array}{l}\bar{\vdots} \\
\vdots \\
\vdots \\
\vdots \\
\vdots \\
\vdots\end{array}$ \\
\hline & $5 \%$ & Is & 86 & 53 & 27 & $\$ 9$ & 85 & 24 & 109 & 70 & 30 & 101 & 71 & 20 & 35 & $\therefore$ & in \\
\hline
\end{tabular}

\begin{tabular}{|c|c|c|c|c|c|c|c|c|c|c|c|c|c|c|c|c|c|}
\hline \multirow[b]{2}{*}{$\frac{\grave{E}}{E}$} & \multicolumn{2}{|c|}{ ruls. } & \multicolumn{3}{|c|}{ Auguet:. } & \multicolumn{3}{|c|}{ September. } & \multicolumn{3}{|c|}{ octciber. } & \multicolumn{3}{|c|}{ Xis? } & \multicolumn{3}{|c|}{ | Irecrube: } \\
\hline & 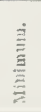 & 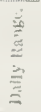 & 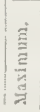 & 音 & 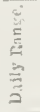 & 莺 & $\frac{\vec{\partial}}{\frac{2}{E}}$ & 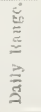 & $\frac{\vec{E}}{\tilde{E}}$ & | & 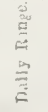 & 竎 & 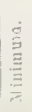 & 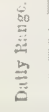 & , & 语 & 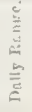 \\
\hline$x$ & $7 r$ & 13 & 32 & 74 & 78 & 33 & $\pi$ & 19 & 37 & 73 & 21 & 90 & os & 22 & 82 & 6.3 & 37 \\
\hline
\end{tabular}

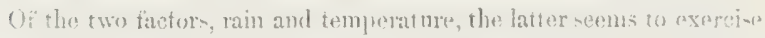
vary litte morlifying influence upon the saroms in the vegetative and wxul life of the plants. It is numly the water that amakefs-

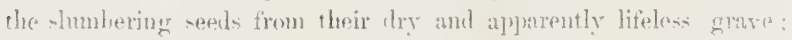
and if the commery is baren and devoid of forest and jumgle, we slall find the cattere of it in the want of rain. Thli- beecomen evident from

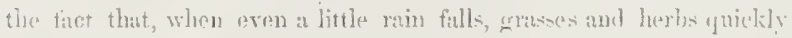

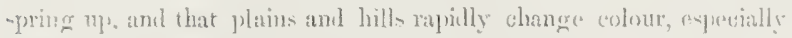
in the trappean area. If dondy wenther follows, sufficient panture in

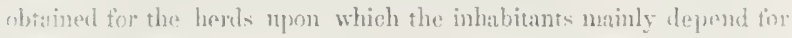
their-ubsi-tence. On the nther hat, in orlinary dry seanons the plainresmble deserts. Heary and iscrifted wer them by the wind often intes torms initating the dumes of seat eansts, and the country seeme (1) affori nowrishment to littl. besides numbers of prickly Juphorbitis. In cases where the rum- are to pantial that lage tracts receive bu it

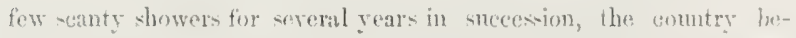
comen all but minhahitec, the prople with their flock being foreed to look out fior subsistence on the imigated lands of Sind or elsewhere.

Thw soil of Cutch is, generally spealking, a light olay, coscred wih a coarve samb from one to four and six inches deep. Six ol aight feet 

below tha =urface, in many places is found a clay of perfectly white colont like lime, and in others we meet with a strong tough yellow an at? mixed with small sones. In the formerwater is said to be found mowi abundant, but not of the best yuality: and in the latter, water of :an axcellent quality is generally sor by penotrating through is thin bed of rock lying loeneath it. In some of the plains netar the hills excellem water is found by cutfing through a sandsone which ofuen lies within four feet of the surfice; and in other plains wells have been sunk sixty or seventy feet deep withont meeting with either water or stone of any kiml. In Varad the soil is more loamy than in any other part af Coteh: and in many instances under the hills throughout the province we meet with a rich soil thickly (overed with a coarse limel of gravel, which seems in some way or other to ail vegretation, Extensive salt wastes, which are not uncommon, are frequently nnoruted with a siline covering, and yipld no vegretation.

The fullowing list contains the indigenons plants as well in thow commonly cultivated. No mention is andde of the unmerous ypecie-

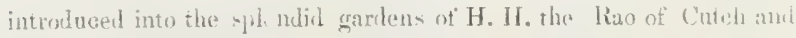
of the Yolifical Agent residing in Bhuj. The geograthlical di-tribution 1 ha been adiled to each pecion in order in fucilitate the formation of a greneral idea as to the origin and composition of the flom aml if present relations to the regetation of the neighlumbiner conutries.

In this place I should like to expres my -incerest thanks to H. H. the Ran of Cutch, to (bol. Abme, the Politieal Agent, to the Drwan Saheb, Mr. Chunilal Sarabhai, and to Mr. K. II. Kotwal, the ('onmi-ioner of l'olice, for the Kind ansistances they gave as thronghin th cur stay in lintel.

1. Mlagnabiacer-

(1) Michelia elsmpaca, L,-Cultivatel : Howers: A pr.-Sept.--3arat.

2. Anonacen-

(2) Polyalthia longifolia. Benth and Hook--Not indigcrous; Horrers Apre-Aray-Ceylon.

(3) Inoura squamosa, L.-Cultivated.-West Indies.

(4) Anona reticulata, L.-Cultivated.-West Indies.

3. Menispermacer-

(5) Tinospora cordifolia, Miers-Konkan, Deccau, S. M. Count2y.

2. The respective notes bave been gathered from the more recent floristic works in Indlian plants, especinlly Th. Cooke and J, D. Hookor. 

(ii) Cocculus villosus, DC.-Fot common; Howers: J)ec--Cinjarat, Decean. Konkan.

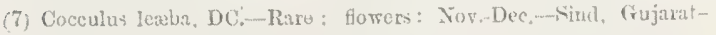
Afghanistan, Arabia, Africa.

(5) Sitphania bernandifolia, Walp,-lare-Decens, Lonkan, KararaMalaza. Tropical Afriea and Australia.

*. Vyminharacer-

(9) Nymphrea lotus, I.-Very rare; culciated in the Bombay l'resi. Aency-Ifrioa, ILungary, Jara, Philippines.

(10) Jymplaxa stellata. Willd.-Thare; cultivated in the Brmbay Jresidency-Afriea.

$\therefore F$ w

(11) Fumaria parribnz, Lam--In cultirated fichls: forrer : Dec.-o) an.sind, Decern.

$\therefore$ Cruciftri-

(12) Farsetia jacquemontii, Hook. f. and Thoms.-Pretty common : Howers : Dec.-Sind, X. India-Afgharistan, Beluchistan.

(13) Moricandia tortuma, Mook. f. and Thoms.- Rare; flowers: Dcesind.

i. Cappritucers-

(14) Cleome monophylla, I.-Fare : Howers: Nor.-1)ec-- injarat, Decen, S. MI. Country-Tropical Africa.

(15) Cleome papillosit, Steud-Flowers: August-Dec--Sind-Arabia. Abrssinia, Nubia, Kiordofan.

(16) Clcome stocksiana, Boiss,-Flowers: Oct--Nov,-Sind-Beluchistan.

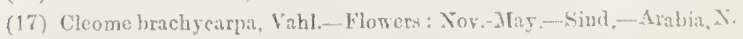
Africa.

(1*) Fynandropsis pentaphylla, DC.-Common in wavte places; flowers: June-Sept,-Gujarat, Decetn, S. M. Country.

(19) Mrzua oralifolia, Cambes.-Rare, in hedges; flowers: Nov.-March.Gujarat, Decean, Konkan, S. II. Country.

(20) Candaba indica, Litm.-Rare, in hedges; fowers: Yov.-MarchGujarat, Deecan, Konkan, S. MT. Country.

(21) Capparis spinosn, Is-- Yot common ; Bowers : Jec,-Mareh.-Sind. Deccan, Konkan-N. Africa, Mediterancan region of Emope, Australia.

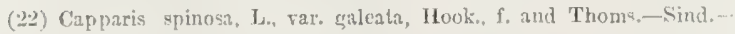
Arabia, 1. Africe

(23) Capparis aphylla, Roth.-Very common; flowers: Fob.-Alarch.Sind, Gujarat, Deccar-Arabia, N. Tropical Africa, Figypt.

(31) Capporis sepiaria, L.-Tare; flores's: Feh,-Mareh.-Decenn, Kanara.

(35) Capparis borrida, L. f.-Not common; flowers: Nor.-tpr.-.sind, Deccan, Konkan, S. M. Country. 

8. Revelucers-

$\left(2_{i j}\right)$ Reseda pruinosa, Welile,-Hare,-Flomers : March.--Sind.

(27) Resela ancheri, Boiss--Kare.-Sind.

1) Tiolacer-

(23) Vinla stocksii. Bois:-Elowers: July-August.-Sind, (injum:Beluchistan, Afghanistan.

in. Pabugabacen-

(2:!) I'nlggala erioptom. I)(:-O) dry cultivated ground.-Sind, fi:: a: Deccan, Konkun-Tropical A-ia and Africa.

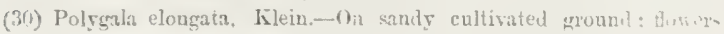
Aug.-Dec-Konkan, lianara, s. MI. Country.

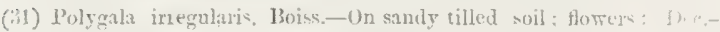
Sind, Gajarat-Beluchistan, Arabia, horlofan.

11. Caryophinllaceie-

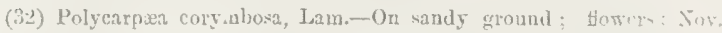
Feb,- Sind, Jecean, Konkan, s. 31. Country-Tropical Lsia. Ifrica, America, A uitralia.

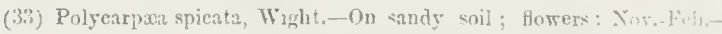
Sind, (Rujarat-Arabia, Abyssinia, Egypt.

12. Porfulacacer:-

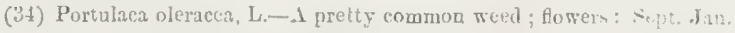
-Throughout the Presidency.

(35) Portulaca quadrifida, L,-Common; flowers: Sepr...Tan,_-ind, Deccan, S, MI. Country.

(36) Portulaca tabcrosa, Roxb,-On sandy soil, less common that the foregoing specie:; flomers: Lug, -Sept.-sind.

13. Tamaricacere-

(37) Tamarix diniea, laxb.-In river beds; flowers: Nox.-3) : Throughont the liresidency-Afghanistan, Assam, Borreo.

(3x) Tamarix ericoiries, Rottl.-Rirer than T lioica; forter: Dos. Jan,-Ginn, fiujurat, Decean, Konkan, Kanara,

14. Elnlinacer-

(39) Bergia odorata, irdgem.-Flowers: Oct.-Doc.-Sind, (xujurat.

15. Malraces-

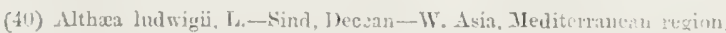
S. Ifrica.

(11) Malsa parriflora, L. - Lloout human habitations: fforry : I) c, Feb_-sind, Deccan -W. Asia, N. Africa, Europe.

(42) Sida Feronicifolia, Lam.-Verf common on sandy enit: foutrexs: Oct.-J)ec.-Sint. (iujari, Konkau, S. II. Country-Tmpical and subtropical regions of the world.

(43) Sida spinosa, L.-Flowers : Oct,-Dec, -Sind, (rujarat, Deccun, Konkan-T'ropical and sulutropical regions of the world. 

(1) Sidi rbumbifolia, var. retusa, Ifaster's-Tery common : bower Oct.-.Tan.--Ginjarat, Decenn, Konakan, ‥ Ml. Country.

(fi) Alutilon indicum, Sweet,-Yery common: fowern: Oet.-.Jan.-Siml, Decen, Konkan, S. MI. Country.

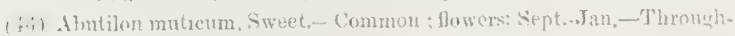
out the Presirtency.

(+i) Abutilou grareolens, W. \& A.- Sot common: flowers: Nox.Jan,-... Sind-From tropical Afrjeil (1) Qucexlshant.

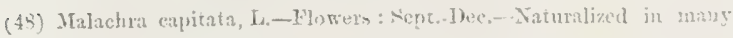
places in India.

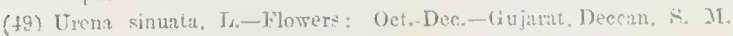
Countrs-11l tropical legions.

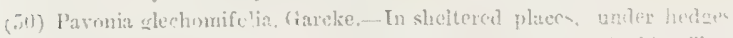

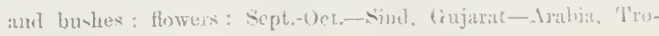
pieal Afriea.

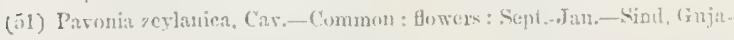
rat, Decean-Wropical Africa, Mauritius, Coylon.

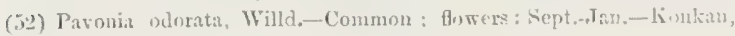
S. M. Country-Tropieal Africa, Ceylon.

(ii) Hibiscus trionum, L.-Floners: Sept.-.Jan,-Sind, Deccu, KonkiuSinuthern kiurope, Tropici of the Uh? World.

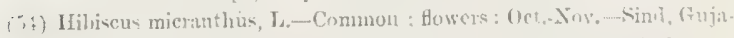
rat, Deccan, Konkan, S. MT. Commtry-Tropical If ica, Cey]nu.

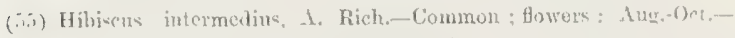
Sink, fujarat-trabia, Tropical Africa.

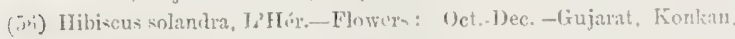
S. Mr. Country-Asia, tropical Ifrica.

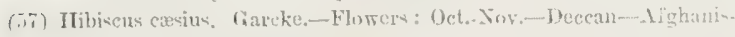
tал, ৯. Anstralia, ‥ Africa.

(5s) Hibjecus punctalas, Dalz,_Flowers: Sept,--Jan.

(59) Hihisens coenlentris, L.-Cultivated.

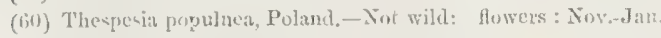

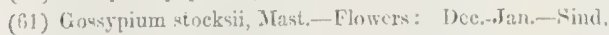

(fiz) Gos-ypium herbaceun, I. var.

(fi:3) Gossypium arbore.1m, I.

16. Sterculincer:-

(ii1) ILelhania tomentusa, Stocks.-(iujarat, Sind.

(bta.) Waltheria indica. L.-Not common; flowers : Sept.-()et.Decean. S. M. Country.-Warmer regions of the werld.

17. Tiliacea.-

(65) frewia populifolia. Vahl--Common ; fowers: Sept...öor-Cinct S. IT. Country-Beluchistan, Afghanistau. S. Yersia, Alabia Trnpical Airica, Mauritius, Ceylon.

(bij) Grewia villosa, Willd.-Common-Sind, fiujarat, Uecean, Konkan. 

(67) Triumfetta rhomboidea. Jacquin.-Common; flowers: Sept...Jan .Decean, Konkan, S. M. Country, Kanara-China, Malaya, Tropical Africa.

(68) Triumfetta rotundifolia, Lam.-Commnn; flowers: Aug.-Sept.Gujarat, Deecan, Konkan.

(69) Corchorus olitorius, L-Common; forvexs: Sept.-Sind, Grujarat Decean, Konkan, S. MI. Country - All tropical regions.

(70) Corchorus antichorus, Raensch.-Common; flowers: Sept.-NovSind, Gujarnt, Deccan-Afghanistan, Arabia, Tropical Africa, Cape le Verde Islands.

(71) Corchorus acutangulus, Lam.--Flowers: Sept.-Uet,-Sind, (rujarat, Konkan-Ceslon.

18. Malpighiacee-

(72) Hiptage martablota, Grertn. ${ }^{1}$-Decon, Konkan, Kanara-Ceylou, Jara, China.

19. Zygophyllacec.-

(73) Tribulus terrestris, L.-Common ; flower: : Augg,-Oct-Dind, Gujarat. Decean, S. MT. Country.

(74) Tribulus alatus, Delile-Flowers: oet.-Dec-Sind-Arabia, N. Africis.

(75) Sectzenia orientalis, Dene.-Sind-Arabia, Afriea.

(76) Peganum harmala, $H_{\text {, }}$-Flomers : Jor.Dec-Sind, Decean, Konkan-Soongaria, Arabia, 1 . Africa, Hungary, Spair.

(77) Zygophyllum simplex, L.-Flowers: Nov.-Jan.-Sind-Arabia. Western Asia, Tropical Africa.

(78) Fagonia cretica, L.-Tery common : thwers: Oet.-Jan.-Sind. Decean.

20. Geraniacee.-

(79) Monsnuia senegalensis, Guill.-P'retty common; flowers: $\Lambda$ ug. Oet,-Sind, Gujarat, Decean-Bcluchistan, Arabis. Senegambia.

(80) Oralis cornicnlate, I, -Flowers : ()et.-June-Cosmopolitan.

21. Rutacea-

(81) Murraya konigii, Spreng--In gardens-J)ecean, Konkan, Kanara, S. M. Country-Ceylon.

(82) Citrus medica, IA, var. medica. Citron,-In gariens.

(83) . . . L., var. limetta, Sweet Lime-In gardens.

(84) " " . L., var. acida, Sour Lime.-In gardens.

(85) Citrus aurantium, L.--Orange. In gardens.

(86) Citrus decumana, Murr-Pammelo. In gardens.

(87) Foronia elephantum, Corr-In gardens-Jara, Ceylon.

22. Simanitaces-

(88) Balanites roxburghii, Planch.-Common ; Dowers : March-MayGujarat, Deecan, Konkan, S. M. Country-Drier parts of India.

1. I have not seen this plant; I give it ore the autbority of Murrey. 

2.). Burzerares.-

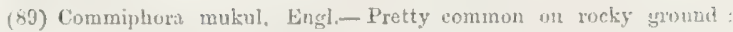
flowers: Dec-spril,-,ind, Decen, Rajuutana, Arabia, Heluchis. t.an.

24. Heliacer:-

(9(1) Melia azedarach, I,--In gardons-Persia, China.

(91) Azadiachta imlion, A. Jus4, - In gardens.

25. Celastraerm.-

(4\$) Gymnomporia moutana, lionth-1)n rocky ground, nol common; fowers: Oct.-Nor, - Sind, Gujarat, Decean, Konkiu, KanaraCentral, South-westen and Sorth-mesterin parts of India, Af. ghanistan, Central Africa. Malaya, Anstralia.

¿ii. Rhamnacear,-

(93) Zizyphus jujuba, Lamk.-Very common : Howors: Sept.-Oct.Throughont Intia,-Afghanistan. Ceylon. Chiua, Bustraliat. Africin.

(9ł) Zizyphus roumdifolia, Lamk.-Not eommon ; flowers : Nept.-Oct.(xujarat, S. M. Country, Yunjal, Westen l'eninsula, l'ersia.

(15) Zixrphus onoplia. Alill,-Not common; towers: Sept.-0et:--.1)ecean Koukan, Kanaliı, S. M. Country-Hiotter parts of India, 'Tropical Asia, Austrilia.

27. Vitacer'-

(!G) Vitis tritolia, I. - Yot common; Howers: Aug,-Deccan, Konkan. Kanara, A. II. Country-throughout India, Ceylon, Malacca.

2x. Sapindaer.--

(97) Sapindus lamifolius, Vahl.-Cultivated; Horrers: ()ct.-bec.-Indi

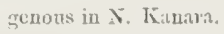

2 ?!. Anacardiacer.

(98) Rhus mysorensis. Heyno,- Not common, on stony dry slopes; Alowers: June-July-Sind, Deccan, S. M. Country-Throughont Intiat.

99) Jangifera indiea, 1, - - inltixuted ; flowers: Jan.-31arch.

iii. Moringacea.-

(14ki) Moringa pterygosperma, Gartn,-Cultivated, near villages in tilled snil--Indigcnous on the W. Himalay and in Oudh.

:1. Leguminosec-

(101) Crotalaria burlia, Ham,-Common in sandy places ; Howers: Dec. Feb.-Sind, (injarat-N. W. India, Afghanistan, Beluchistan.

(102) Crotalariat retusa, L.-Common; Howers: Sep.-Jau.-Decean, Komkan, S. M. Country-Throughout India, Ceylon, China, Malaya X. Australin, Tropical Africa.

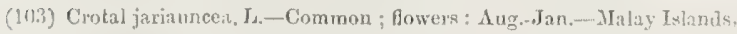
Australia. 

(101) Crotalarin medicaginea, Lam. var. negrlecta, Baker.-Sint, (rujarat, Konkan-Tropical India, Ceylon, Mahya, Afghanistan, China, Anstralia.

(105) Crotalaria notonii, W. and 1.-On sandy wil-Gujarat, Nilghiri and l$^{3}$ iney Hills.

(10ii) Telilutus indica, All.-Sind, Decen-Tropical Indix, Furupe S. Persia, Afghanistan.

(11\%) Medicago kativa, L, - Cultivated.

(108) Lotus garcini, DC--Flowers: Sov-Dee,-Sind, Gujarat-l’oxir Yubia.

(10y) Indigofera linifolia, Retz.-Common: Hower : Jug.-Dec--Decean Konkan, S. II. Country-Throughout India, Ueylon, Ifghanistan, Abysuinis N. Anstralia.

(110) Indigofera enneapbylla, I.-Common : Ilomers : Aug.-1)ec-Cinjarat, Decan, Konkan, S. M. Conntry-l'lains of Imlia. Ceylon Angoli, Malay Islands, X. Australia.

(111) Indigofera anahirptista, Stcuh.-Flowers: Lin.- Fov.-Sind-Punjah, If ghianiotan, Arnbiat.

(112) Indigoferat paucifolia, Delite-Common; flowers: All the yuarGujarat, Sim-Plains of India, Ceylon, Beluchistan, Arabia Java, Tropical Ifriea.

(11ii) Indigofera trifoliata, J.-Xint common; Howers: Sept. SovGujatat, Decean. S. M. Country, Konkan-Thronghout India Ceflon, Java, China, P'hilippines, X. Australia.

(214) Indigofera articulata, Goilan.-Flowers: Sovo-Dec.-Sind, Deecan-Arabia, Iiggpi, Abysinial.

(11j) Indirofera tenuifolia, Rottl,-Flowers: Oct.-Nov-Sind, fámanat. S. M. Country, Konkan-Wextern Peninstala, Ceylon.

(116) Indigofcri parvillora, Heyne-Llare; Llowers: Nov.-Dec. -Konlan, S. If. Country-Wosteru Peninalua, Carnatic, Aratia, Tropical Lfriea, D. Australia.

(117) Indigofera tinctoria, J.-Fot common; Howers: Scpt.-1)ec.

(118) Indigofera hirsuta, I.-Fot conmon; flowers: Sept.-Dec.-Konkari, S. II. Country-Plains of India-Ceylon, 'Tropical Africa anul America, Jara, Philippines, N. Australia.

(113) Tephrosia tenuis, Wall.-Flowers : Sept.-Nov,-Sind. Deccan. Konka1, S. M. Country-Punjab, Burma, Lacendives.

(120) Tephrosia purpurea. Pers.-In sandy places; flowers : Sept.-Dec.Gujarat, Itecean. Konkan, S. IT. Country.

(121) Tepluosiat villosa, I'ers,-var. incana, Baker: Common in sandy places ; flewers : Sept.-Dee,-- (rujarat, S. M. Country.

(122) 'Tephrosia senticosa, 1'ers-In kanily llaces: flowers: Rept.-JecDeceun, Konkan, Westerfi Peninsula-Burma, Ceylon.

(123) Sesbania agyptiaca, Poir. var. hicolor, Wh and A.-Flewers: sept.-1)ec. 



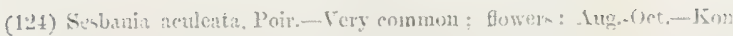
kan. Heecan-Tropies of the (old Worthl.

(125) T'apernich utmmularia, DC,-Flowers: J)e, - Sind, Gujamt, Jeecat - Punjah. Af phanisan.

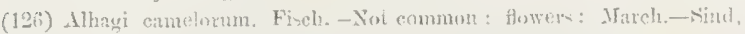
Gujamt, S. M. Country. X. ami X..W. Povinces-JBeluchiscar. Egyt, Arabis.

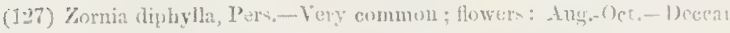
S. M. Country.

(128) Als-iearqus vaginalis. 1)C. flowers: Oct.-Ans.-(xujaraz, Denean Koukan, throughout India-Cerlnu, Afghanistan, Trupte of the ())d IVorld.

(129) Alywierpus longifolius, W. aud A.-Howers : Sept,-frujatat. Jjeccan. Konkan, I'lains of India.

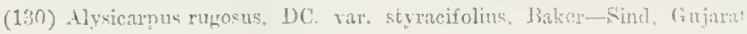
Fonkan, throughont India-Ceylon.

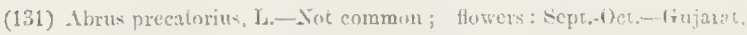
Deccan, Konkan, Kanara.-India. Ceylon. thronghont the T'ropics.

(132) buter frondosa, Koenig,-Rare; flowers : Feh. March-L (xujarat. Decean, Konkan-India, Ceylon.

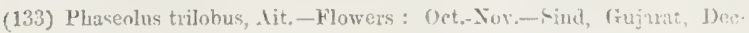
can, Konkan.

(134) Phascolus vulgaris, 1.-Cultivated-S. Amenica.

(135) Phascolus mungo, L., var. roxburghi, Prain-Cultisated in the rainy season.

(136) Phaseolus aconitifolius, Jacquin.-Cultivated in the rainy seacon.

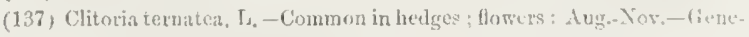
xally in the 'l'ropies.

(138) Dolichns lablab, L,-Cultivated-Tropics of the Old World.

(139) Rhynchosia misima, DC:-Common in bedges-tlowers : Aug.-Jan.Sinu, Gujamt, Deccon, Konksn, S. M. Conutry, India-Ceylon. throughout the Tropies. Care, Uuited s'tates.

(140) Pongamia glalira, Vent.-Planted.

(141) Cajanus indicus, Speng.-Cnltivated-Tropical Africa.

(14:2) Cicer arictium, L, - Very little grown. Cold weather:

(143) Caxalpinia bontucella, Fleming.-Not conmon; forerw: Aus.Sept.-Simd, I)ccan, Kanara-India, throughont the Tropic:.

(141) Caralpinia pulcherima, Swart:-Cultivated.

(145) Poinciani clata, 1.-Planted.

(14i) Peinciana regia, Bojer.-Planted,-Mardagascar.

(147) Parkinsonia aculeata, L-Naturalized-Tropical America.

(148) Cassia oecidentalis, L.-Not common ; Howers : Aug. Oct.-Through out India and the Tropies. 

(1.45) Cassia sophora, J.-Wot common; forer: Augr-Nor.-India, most Tropicul Countries.

(150) Cassia tora, I.-Not common; flowers : Sept.-Dec.-Mndia, gencually throughout the Tropics.

(151) Cassia auriculata, L.-Gujarat, Decen, Konkan, S. Mr. Country, Western P'euinsula, Central Provinces-Coylon.

(152) Casia obtusa, Roxb.-Not common: forrer: Nor-Feb-Sind, Gujarat, Decear, S. II. Country.

(15i) Cassia holosericen, Fresen-Flowers: Now-Jan-_hind-Arabia, Tropical Arici.

(154) Cassia punila, Lamk,-Tery ermmon: flowers: Aug.-Sept.Decean, S. M. Country, Kanara,-Tropical Asia and Australia.

(15i) Tamarindus indica, IL.-Not common, about villages, cultivaterl.

(15i) Bauhinia racemosa, Lank--Rare: forer: Irarch-Junc-J)eccun. Konkan, Imulia-Ceylon, China. 'limor.

(1ii7) Prosopis spiciger.., L.-Vury common; thowers: Dec.-Alareh-Find. Gujarat, Indin-Behehistan, Af ferlanistim, Persiat.

(15s) Dichrostachys cinerea, IV. and A.- Kare, on diy stomy ground: flowers: Oct,-Nor:-Decem, S. M. Cumtry, Kanara, Iudia-Ceylon. Malay Islinds, N. Australia.

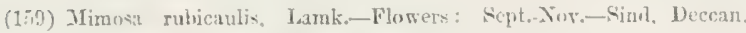
thronghout India, Ifghaistan.

(16il) Mimmat hamata, Willd-Common ; Howers: Sept.-Uct.-Gajarat, Decan, IJunkan, S. M. Country, Westent Peninsula.

(I61) Acacia aribica. Wilh.-Very common; formers: Tuly-Veh.-TnulinCeylon, Arabia, Enypt, Tropical Lfrica, Katil.

(1ti2) Acacia farnesiana, Willt.-Not indigeuous ; Howers: Sept.-MarchGiod, Decean, throughout the Tropics.

(10i:) Acacia eburuea, Willd.-Pretty common ; flowers: Nox-Feb.Sind, S. AI. Country. India-Cerlon, Arabia, Ifyhanistan.

(16t) Acacia jacquemonti, Jentb,-Rare; flowers: Jan,-May-Sind, Grujarat, Punjab, Rajputana.

(165) Acteia lencophloce, Willd,-Common ; flowers": Dec.-Fob,-Deccan, 5. M. Country.

(16.3) Acacia catechu, Willa., var. sundra P'rain.-Pretty common in hushy Jocalities-frujarat, Deccan, Konkan, S. M. Country.

(167) Albizzia lebbek, Benth.-Plantcel in gardens and near well; flowers: March-May-Tropical and subtropical Asia and Ifrec.

:3. Hosacrin.-

(168) Potentilla supina, L.-Flowers: Oct.-Nov-Sind, Fujarat, IndiaAfghanistan, Mllantic, Ni. Africa, N. Asia.

23. Saxifrugacen:-

(169) Vahlia viscusa, Roxb.-Flowers: Dec.-Jan,_Sind, Gujarat, Konkan, India-Persia, Egypt, Tropical Africa. 

34. Haloragidacer-

(170) Iyriophyllum intermedium, DC:-In ponds-Deccan, S. M. Commtry, Kinara. Western Peninsula-Malaya, Lustralia, New Zcalaud. S. America.

3.5. Rhisophoracer-

(171) Rhizophora mucruata, hamk-In salt-marshes and tidal creek-

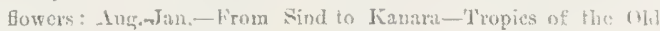
World and Australia.

(172) Rhizophora conjugata. I, - Less common than the ineconime species, in the same loralities; flowers: Aug...Tan.

(173) Iruguiera gymorhiza, Jamk,-In salt marshes along the coast.

30. Myrtacce.-

(171) Eugenia jambolana, Lamk.-In gardens and near wells : flower': March-Way-Throughout the Presidency, but not in simet. Indii genexally-Ceylon, Malaya, Australia.

(175) Pxidium guyara, 1.-Cultivated-Mrexico.

i7. Lythracea-

(176) Ammanaia baceifera, J.-Wiowers: Nor.-1)ee--Sind, ('xujuxat, H)e

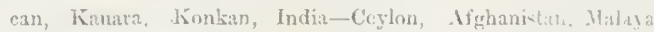
China, Iustralit, Tropieal Ifrica.

(177) Ammannia silicifolia, Mnnti.-Flowers: Toץ,--Sind, Decenn. S. M. Comntry, Konkan, throughout India-T'rnpical Afticik.

(178) Lawsonia inermis, L.-Girowing wilk and cultivated.

(179) P'unica mranatum, 1, - (inltisated-Wild in P'orsia, 1Bcluphi-ian, Afuhatuistan.

\section{Cucurbitaceo-}

(180) Trichosinthes angruina, $L_{n}-$ Cultivated.

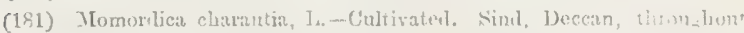
India-Ceylon. IIalay, China, Tropieul Ifrica.

(182) Momordica baleanina, L.-Flowers: Noy,-Der,-sini, Jeccin: frujurat, Panjai, X.-W. Provinces-Malaya. Ausiralia. It. A-ia, Africa.

(18:3) Lufia agyptiaca, Mill.-In gartens.

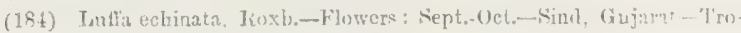
pical Africa.

(185) Cueumix trigonus, Roxb-Common; Homers: Ango-Der, Sint. 1)ecarı, all oser Imein-Ceylon, Afghanistan, Persin. Malaya. r. Arstializ.

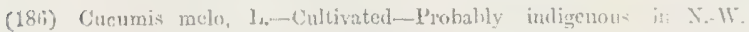
India, Beluchistan and Tronpical Africa.

(187) Cucumis melo, I. พar. agrestic, Naur,--Common-Gind, Jjecean s.. M. Country.

188) Bryonopsis lacininse, Noud.- Fot common, in hedges: flomor 

Aug-Oct-Decean, S. M. Conntry, throughout Tndia-Ceylon. T'ropical Africa, Manritins, Malaya, Australia.

(1sti") Citrullus colocynthin, Schrader.-Pretty commou on sandy grouni -Sind, Gujarat, Jecean, Konkan, all over India-Coylon, Alabia, WT. Asiz, Africa, Spain.

(190) Citrullus vulgaris, Schral,-Cultirated-Mila in Tropical Ifrica.

(191) Ceccinia indica. IV, and 1. - Fory common in herleges ; funers Almost throughont the wholo year-sind. Gujant, A. XI. Comstry. Decan, a!l oyes India-Ceylon, Malaya, Tropical Africa.

(1:2) Melothria materi-patana, Cognniaux-Common inh edges and bushes-Sind, Gxujuat, Deccan, Ínnkan, s. M. Country, all over India-Ceylon, Africa, Malaya, Australia.

(19:3) Blastania gareini, Cogniaux.-Not common : fowers: Oet.-Nov:Decean, tiujurat, s. AL. Country.

(194) Coraliocurpus uniga:us, C. B. Clarke-l’retty common-sind, Guja zat, Decom, S. M. Country, Pujab, Westem I'eninsula-Cul.no.

(195) Lageraria rulgaris, Seringe,-Cultivated.-Wild in Malabar. Dohrat Doon-1 byssinia, Mtoluecas.

(196) Cucurbita moschata, Duchesne,-Cultirated.

(197) Cucurbita naxima, Duchesnc.-Cultirated.

(198) Cucurbita jepo, L.-Cultirated.

:39. Cactrecer-

(199) Opuntia thllenii, Haw.-Not common; used for hedgen.

4i. Ficoirlon-

(200) Trianthema monogyna, L. - Very common: Sind, Deccan, all over India-Ceylon, most Tropical Countries.

(201) Trianthema tripuetra, Rottl, \& Willel.-Very enmmon: flowers

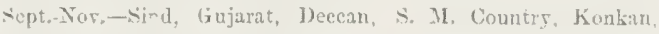
I'unjab, Wr. Peuinsula-Ceylus.

(21i2) Trinthema pertandra, L.-Common: flowers: Oet.-1)ec.-Sind. Deccan, S. M. Country, Konkan, Punjah, X.-W. Provinces, W. Perinsnla-Tropical $A$ frica.

(2113) Orggia decumbens, Forsk, Flowers: Itec-kcb.-Sind, Punjal,. Aysore-Western Acia, Africa.

(204) Mollugo hirta, Thunb.-Lint common, in dried up water holes,Sind, Decoun, S. MI. Country, Konkan, all over India-Cerlon warmer regions of the World.

(205) G̈iscbia pharmatceoides, L.-Rare; flowers : Sept.-Oct,-Sind, Gujaral s. M. Couutry, Konkan, Puxjah, W. Peninala, Ceylon, Boluchis. Lan, Afghanistan, Africa.

11. L'mbolliferce:-

(20ii) Dancus carota, J.-Cultivated.

(207) Coriandrum sativum, L.-Cultivated. 

4:2 Ruliacer, -

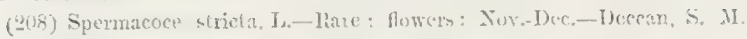
Conntry. Kunkin, India-Ceylen, Tropical tain and Africa.

4:i. Conprositu, -

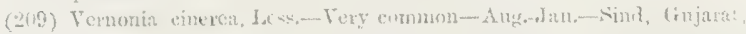

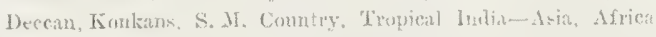
and Lustralin.

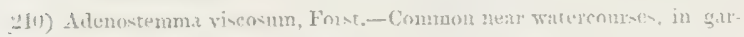
ders and fielits: flomern: Gept.-Feh.-Thecan, Fonkan, S. II. Country, all orer india-Cirylon.

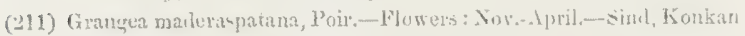

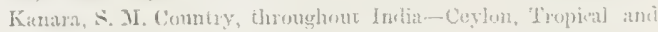
Subtroricale, Asiz anel Africa.

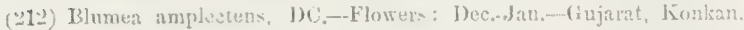
thronghout India-Ceylon.

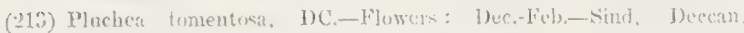
Konkan, Kanam, s. II. Conntry, We-tent Penimsula, Centrat Imlia, Isengal,

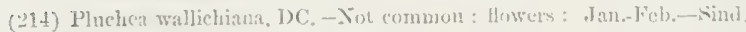
\{rujumt, Punjah-Belnchistan.

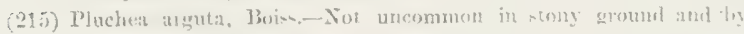

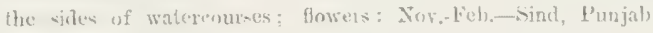
1) luchi-tan.

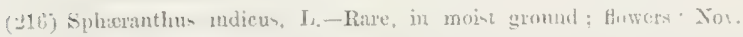
Fol,-Gajarat, Decan, Koukan, S. II. Country, all orer IndiaCeylon, Africa, Maliy Islands, Australia.

(217) Gnapholium luten-allum, 1.-Sind, Decean, Kunkan, hot and warm temperate countries.

(2ts) Gnaphalium indicum, L.-Flewer: Dec-Fub. - hind, 1)ecan, S. IJ. Country--India, Buma, Africa, China, Jipan, Australia.

(219) Inula grantioides. Jois--Common on racky ground; flowers: Nos Jan.-Gind. Beluchintan, Wazari-tan, ‥ E. Arabia.

(220). Vicoa auriculata, Cass,-Flowers: Nor-Feh,-Werean, Konlsan, , A. Cuntry-India, Ceylon.

(221) I'ulicaria angustifola, DC.-Common in sandy soil; flowers: Nor. Tan,-Finel, Fujarnt, Konkan, Wentern P'eninsula, Clueti Yagpur. liengal-13tluchistan.

(22,2) Siegerbeckia orientalis. I.-Flowers: Nos.-Jut.-Docenn, \&. AI. Conntry-Most tropical and subtropical conntries of the work.

(223) Felipta erecta. 1.-Flowers: Now,-Jan.-Sind, 1)ecan, Kunkan, Wer. tern Peninsula, Contral India, P'unjab, Bengal-Burma, Ceylon, Malnga, everywhere in marm climates,

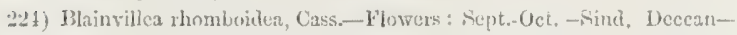
India, Ceylon, Java, America, Australia. 

(225) Bidons pilosa, L.-Flowers : Nmost all the year-Ucecan-Most warm countries.

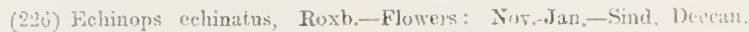
Konkan, S. M. Country, all over Indial.

(227) Volutalla divaricata. Benth, \& Hook. f.-Flowers: Nov̌-1)ecSind, Grujarat, Deecan, Konkan, Wostern, Central, and Sonthern India-Beluchistan, Afghlaistan.

(2:8) Jicoma tomentusa, Cass.-Flowers: Nov.-Mareh-Sind, Gujarat Deccar, S. M. Country-W. Peniusula, N.-W. Provinees, Tropical Arrica.

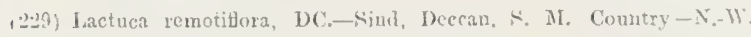
Provinces, Ar,bia.

(230) Launæa pinnatifida, Cass.-Common; Dec.-Jan.-Sinu, Giujumt, Konkan-India, Ceylon, Mauritius, Fgypt, H. Afriea.

14. Gooderiesceu.-

(2:31) Sexvola kanigij, Vahl,-On the sea-const; Howers: Juiy-AugSind, Kunkan-Sen-shores of India, Ceylon, 'Tropical E. A-iz Anstralia, Polynosia.

45. Plumbrgimacer.-

(232) Statice stuchsi, Boiss-Flowers: Dec-feb.-Mint, Gujarat. Belnchistan.

16. Prinulacen:-

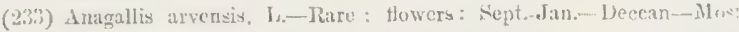
temperate zegions.

47. Myrsinacer.-

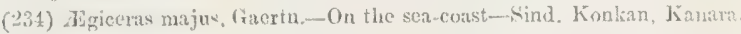
48. Sapotacre.-

(235) Jimuogps elengi, L.-Cultivatet ; flowers: Dec.-March.-Fajarat, Decean, honkan. Kanara-Western l'enimsula-Coylon. Mralaya.

(236) Mimusops hexardra, Roxb.-Cultivated in gardens and nenr wells fluwers : Sept.-Dec.-thinjarat, Deecan, Konkan, S. M. Country--. IT Peninsula, Ceylon.

19. Oleacerr--

(237) Jasminum sambac, Ait-Cultivated.

50. Salvadorucece.-

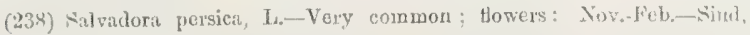
Gujarat, Decan, Konkan, Kanara, S. M. Country-Drier xesinus of Iudia, Ceylon, dry parts of W. Asia, Egypt, Abyscinia.

(2u9) Salvadon oleoides, Decaisne-Common; Hower: Dec-Mirch-.Sind. (rujarat, Punjab, Rajputan, Aden.

5.1. Aprocynacen.-

(240) Verium otorum, Soland.-(Grown in gartens, foumd at an eserpe in some places; flowers: All the year.-Indigenons in the Wort. ern Himalaya and Nepal. 

(211) Plumeria antifolia, Poir--Cultivalen,-Indigenous in Trontas Anericer.

52. Asclepiulacer.-

(242) Peiphloca aphyllit, Vecaisue,-Common on rocky ground, sini, Panjab--Afghanistan, 13eluchistan, Persia, Arabir. Hegpt.

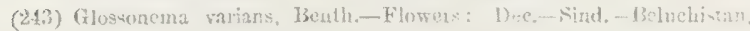
l'crsia.

(244) Calotropis procerz, R. Br.-Tery eommole ; Homers: Jec. Jan.-

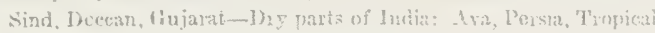
Ifrica

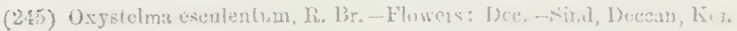
kan, Fanara, s. M. Country. -India, Cen]on, bava.

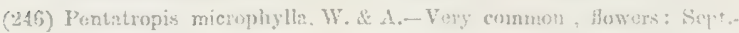

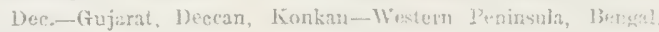
l'emu, Coyion.

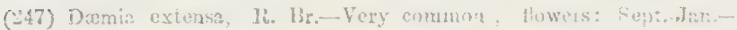
Sind. (1) uiarat, 1) tecan, Kentem, \&. H. Comntiy.

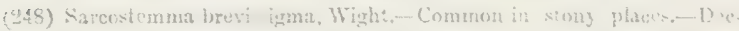

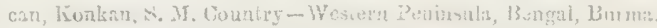

(249) Leptatenia reticul.ts, W. \& A.-Jane, (sujaral, l)cecan, Konkan

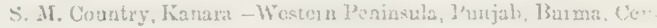
lon, Singapore, Asu.

(250) Leptadena spatiun. Wight,--Wery common ; flowors : j)ec, bin,

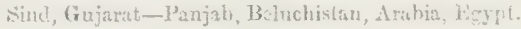

53. Gertianaceor.-

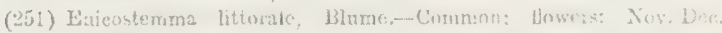

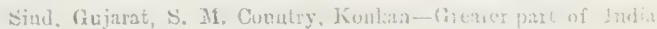
Coyloa, Mralıya, 'l'ropical Ifrica. W. India.

54. Boragìnaccer.-

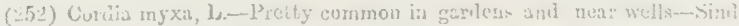

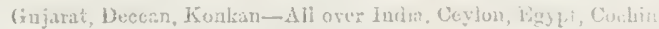
China, Tropical Aushalia.

(25:3) Cordia rothii, Hown. \& Seluult-- Very conimon: flowers: Nox.

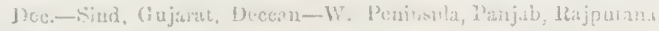

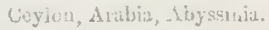

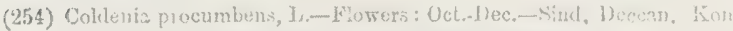
karn, S. Al. Cuuntry, Kanara-'i'tupıs ge nually.

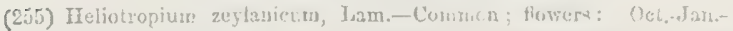
Sind, (Tujarat, S. M. Country, Konkan-Western P'vinisula, I'an. juk, 'leopienl Airiea.

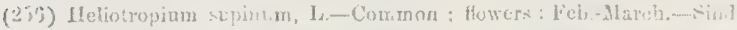

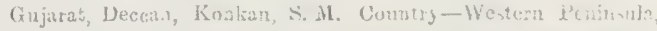

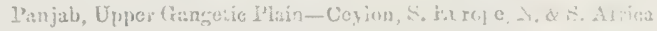
Canary Lshame. 

(357) If liotrotium panicuatum, R. Mr.-Rare-Sind, S. M1. Coantry W. I'enirtsula, Pegu, Ceylon, siam, dustralia.

(2iv) Tricholesma inticum, 1R. Br.-Common; Howers: Sept.-Nov.Decen. Konkan, S. M. Country-Grealer part of India, Coylon. Cubul, Behubi-tan, Persis, Maritius.

(250) Trichodesma reylanicum. 1i. Br.-Common; flofers : Dec.-dan.Gujarat, Deecan, Konkan, S. Mi. Courtry, Kanara.

(26i) Sericostoma pauciflorum, Ntucks,-Flowera: Nov,-Dec.-Find. Gujarat.

(2i1) Amebia hiapidi sima, DC,-sind, Gujaral-Wesiern Egyet. Nubia. 35. Cinnerolvelucere.-

(262) Cuseuta refora. Roxb.-Flowers: Dec-Feb.-Gujarat, $\therefore$ is. Conntry, Kunkan-Throughout India, Coylou, Malaya.

(26.3) Cuscuta chineniv, Lark.-Common; flowers: Ang.-Oct.-DeceanGreater part of India. Ceyluo, Persia, Australia.

\{264) Cressa eretica, L.-Not common ; fowers: Vox,-Jan,-Sind, Cinjarrat, Deecart, Konkan, linara-Warm regions genesally:

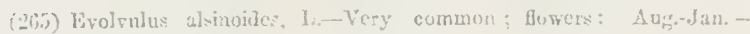
Sinu, (rujarat. Đecean, lionkan-T'ıpical and siabtropical conniries.

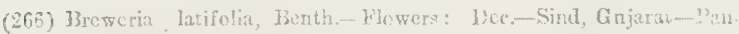
jais, Trupical Aricas.

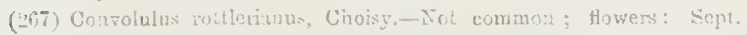

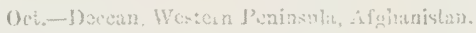

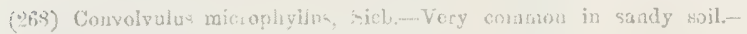
Siad, Gujamal-23:lachistan to Jigypu and Nabia.

(26i!l) Convolrules stomeratus, Choivy.-Floners: Duc.-Wind, GrujaratJanjab, Aschanistan, Jicluchistan, W. Tropical Arica.

(27(1) Convolvulus arvensi:. 1.-Common in cullivated glound f fowers: 1)ac.Pelir.-Xind, Grijant, Jecenr, Kanara-Thrunghout the (พ)?:16.

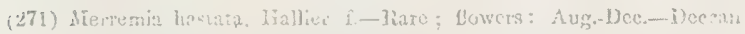

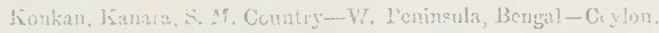
S. L. Asia, Alastailia, "Heppical Africa.

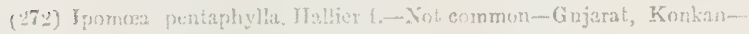

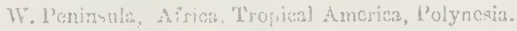

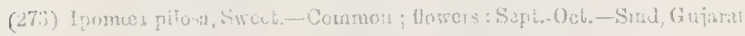

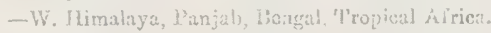

(271) Iromen bilubi, Fur:k.-Émznon on the sandy sea shore.

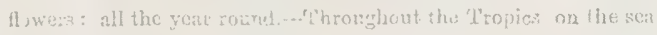
wh rive,

(275) Inomos pessigridiz, $X_{1}$-Very comrnon on sandy ground; flowers:

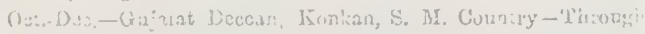
out Indab. Coylon. Mialayn. China, Polymetia, Tropucal Áirica. 

(276) Ipomoxt aruatica, Forsk.-In ponds, zot comrnou; flowers: Nins. April.-Throughont the Presidency-AJ! oper Jnelia. Cuyfon. 'Tropical Asia. Africa. Ausiratia.

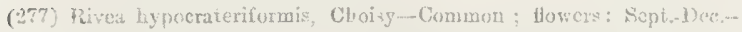
Sind. Uecean, Fonkan-Westerm Peninsula, As:am.

(278) Argyreia speciosa, Sweck, -In garenos; throughout Iadia, Tava.

(To bu conlinzere.) 



\section{$\therefore$}

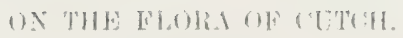

Hi:

l. $1,1,1, \ldots, \cdots$

P.IVT II.

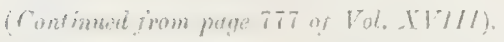

in. Solanitary.-

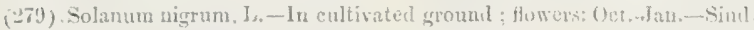

Decean, honkan, \$. M. Country-Temperate and tropical regions of the workl.

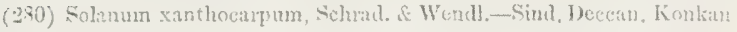

All orer India, Ceylon. S. E. Asia. Malayat, 'T'ropical Australit, l'olynesia.

(281) Solanum indicum, $\mathbf{T}$,-Common.-Decean, Konkan-Tropieal Indì, Ceylon, Malaya, China, Philipprines.

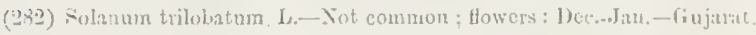
Decean, S. M. Country-W. Peninsula, Ceylon, Mrajay Peninsuli.

(28:3) Salanun tuberosum, L.-Cultiratod-Indiqenous in -I mericit.

(ㄱil) Solanum melongena, L-Cultivated.

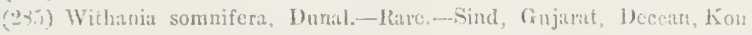
kan-Drier regions of India, Ceylon, Mediterrancan regions. Canares, Cape of liood Hope.

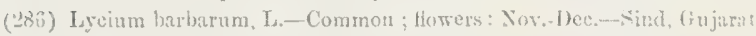
-Panjab, Beluchistan. Af ghanistan, Bersia.

(287) Uatura fastuosa, It-rar. alia, C. B. Clatke-Common : thowers: Sept.-Dec.-I'hroughout the l'residency.

(2X3) Lycopersicum esculentum, Mill.-Cnltivated.-Indigenous in Tropi enl America.

(289) Nicotiana tahaeum, L.-Culisated-I'robably indigcnous in $\mathrm{S}$. Imerica.

(290) Capsicum frutescens, L.-Cultivater.

57. Acroplu uriacer.-

(291) Celsia coromandeliana, Kahl.-Flowers: J:tn.-Ang,-Sind, frujam, Decean, Konkan-All over Iulia, Ceylon, Afghanistan, dra China.

(232) Lisuria ramosissima, Wall,-Dretty common in roeky places,-... 'I'hroughout the Pre-idency - Throughout India, Afghanistan. A va.

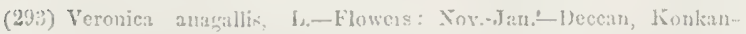
Western l'eninsula, Panjab, hákmir, liengal, Asaln, Bhotan X. Asia, S. Africa, Burope, X. America.

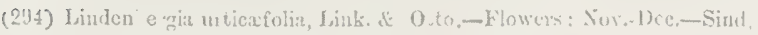
Decean-All orer India, Ifghanistan. 

is. Nevlututherert.-

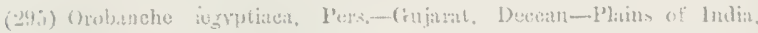
Cintral and Westem Isia.

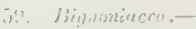

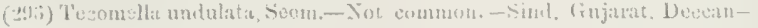

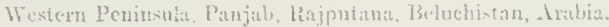

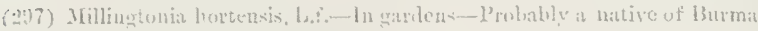
and the Matay - helipelagn.

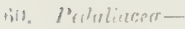

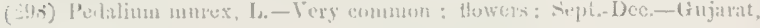
Kisnkan, :. II. Corantry-Intial, Ceyton, Trupieal Afriea.

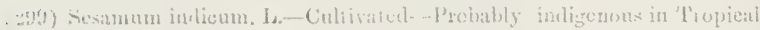
Africal.

ijl. Acinthocese.-

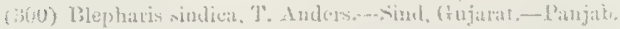

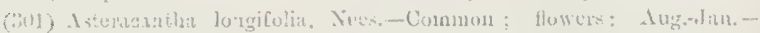

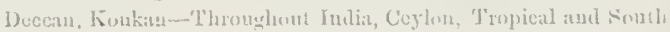
Ifricil.

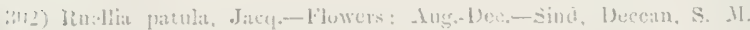

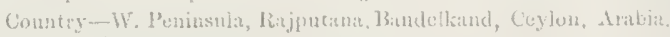
Tropical Africil, Aria.

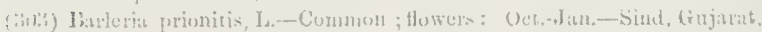

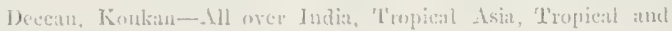
Sunth Africit.

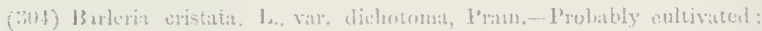
(kuwers: b)ee.

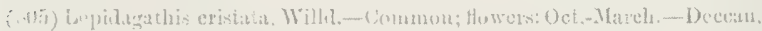

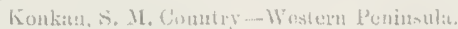

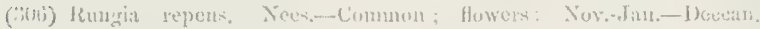

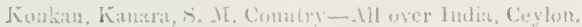

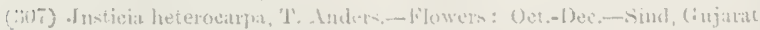
- P'anjilu, Tropisal Irricas.

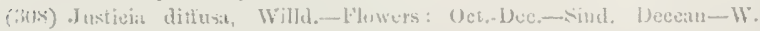

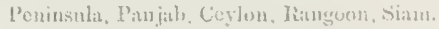

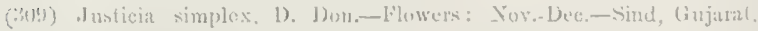
Knnkan. Kanzura, s. M. Country- IV. L'eniusuli, Central India. Kinshur. Bumgal, Abysosinia, Malay lalanris.

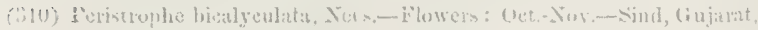

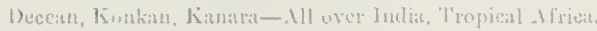

1iz. Verlinuces:--.-

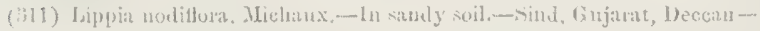
Intia generally. Ceylons. Afrior, most tropionl aut subtropical conufries.

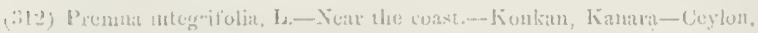
Andaunar, Nicobars, Matityab. 



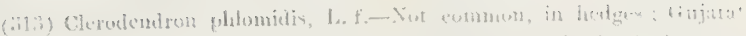

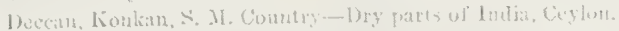

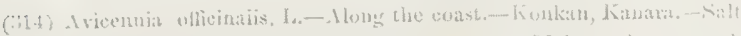

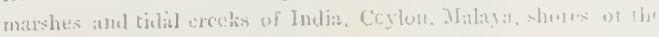
Inlian ant P'acitie Ucerans.

(3). Tabieries.-

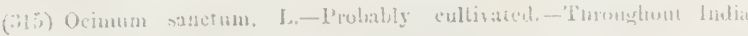
(cultivated). Malizy Archipelikgo, Austalia, Alahlat. W. A-ia.

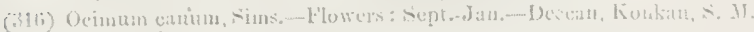

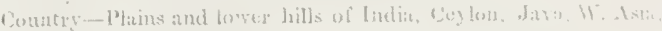
Tropical - triea, Malitgathear.

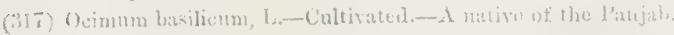

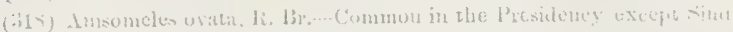

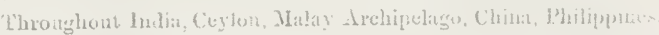

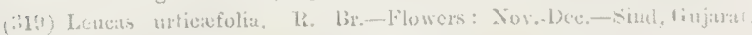

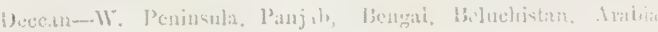
Abysinitia.

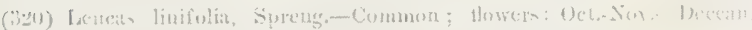

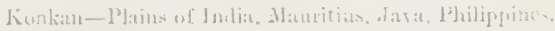

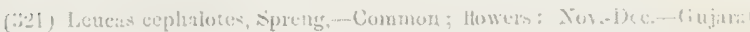

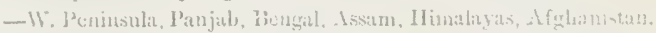

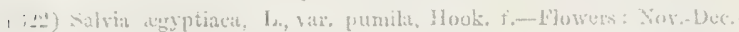

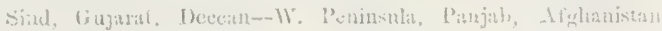
Beluchintule.

it. Sinclegizeseren.

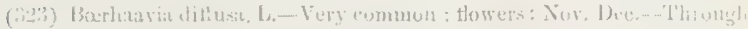

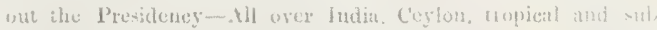
tropical Asia, Ifrica and Anerica.

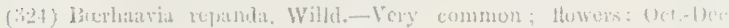
(ixjurat, d)ece:tn, Konkiun-India, Ceylom, Jinluchistith.

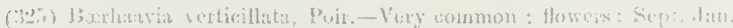

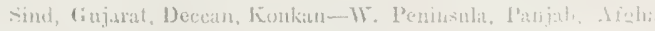

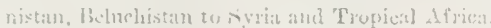

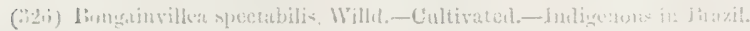

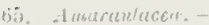

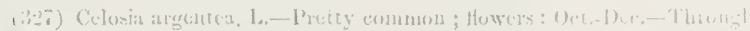

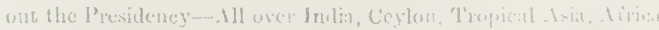

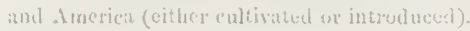

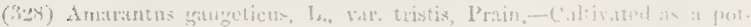
herl.

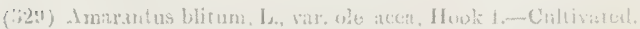

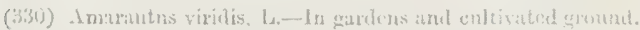

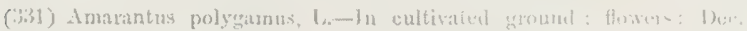
March-lartiat, Ceylun, most hot evuritios. 



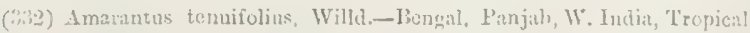
Africa.

(2:3) Wrus javinica, Juss.-Vcry common ; flowers: Sept.-Dec-_Sind, Decenn-W. Peninsula, Contral India, Panjab. Burma, Cerlon, Arabia, Tropieal Africa, Cape de Verde Islands.

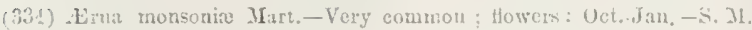
Country-Central India. W. I'eninsula, B'engal, Burma.

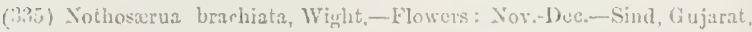
Konkan-Western Peninsula, Burma, Coylon, Manritius, Tropical Ifricil.

(ojsi) Achyranthes aspera, L.-Common: flowers: Nor... Jan.-Sind, Deccan, Konkin -Thronghout, Inda, Ceylon, Tropical Isia, Africn, Australia and America.

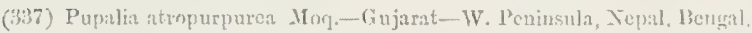
Mializa, Ceylun Java, I'hilippines, \&. Africa.

(338) P'upalia lappacen. Moq. - Plowers: Scept.-Dec.-Sind, Gujart, Decen. S. M. Country-W. Peninsula, Jchar, Panjab. W. Tropical Itymalizy. 'Tropical Asia and Africu.

(33!) - Ilternanthera triandra, Lam,-Duwers: Awg.-Dec.-Throughout the Presillency - All warm coluntrick.

\section{iti. Cheurpotiacet:-}

(340) Atriplex stocksii, Boiss.-Flowers: Dee.-Jan.-Sind, Gujuth.

(341) Salicoruia brachiati, lloxh,-Flowers: Now,-1)ec-Giujuat-bongal. C'eylon.

(312) Suxda fruticosa. Forsk.-Flowors: Now-Dec-Sind, Gujarat - N. W. India Paujab Africa. America.

(343) Suada nudifora, Mog,-Konkan, Orissi-W. Peninsula Ceytou.

(311) Iraloxylon recurvum, Bunge,-Flomers: Nor-Dec-Sind-Coimbatore, Burma Parjab Afghanistan Yunnan.

(345) Salsola fatida, Del-Flowers: Dec-Sind-P'anjab, Beluchistau. Persia, Arabia, N. Africa.

6. Pobygonscer.-

(34b) Pulyonom plebejum, R. Br., Far. indica, Honk, f,-Commun,Decent, Konkan, s. M. Country, Kisma.

(3\$7) Polygumum glabrum, Willd.-Flowers: Oct.Feb.-Sind, Heccan. Konkan, Kanara-All over Indı, Ceylon, Australnsia, China, Ifrica. America.

is. Aristolochincer:-

(348) Aristolochia bracteata, Retz.-Flowers: Sept.-1)ec-CSinel, Gujarat. Deccun, Konkin-1Bcugal, Ceylon, Aribia, Tropical Africa.

50. Euthortinceo,-

(319) Euphorthia nerifulin, Is-Very common on rocky ground; flomers: Mrurch,-Sind, Gujarat-x. WV. Himaliza, Pegu, Burma. 



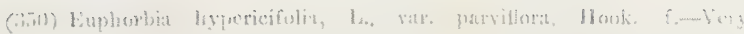

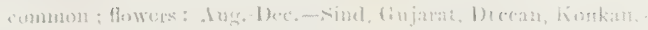

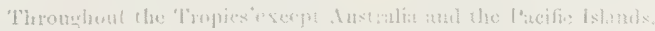

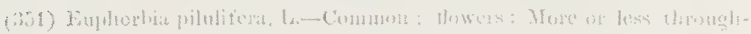

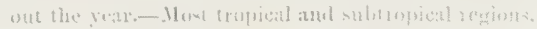

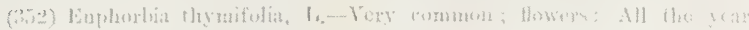

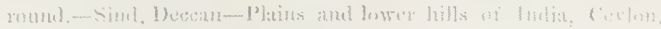
all lant conntries atsept. Instratia.

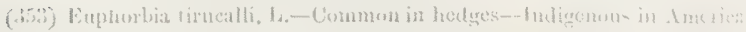

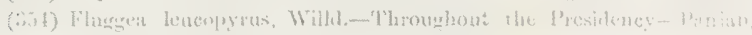

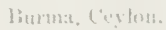

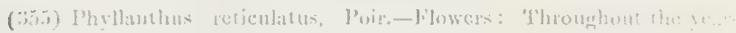

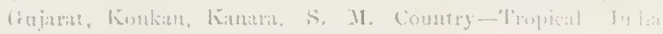
('eykun. Tropical Africat. Chinn. Malay Islauks.

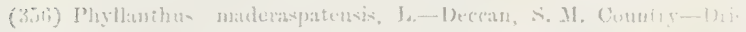

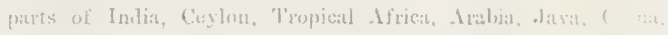
Anstralit.

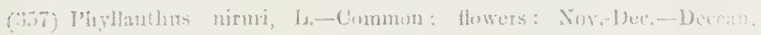

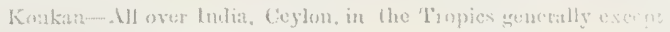
1... thit.

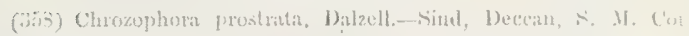
Througlnui india. X. Africis.

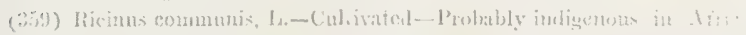

il) Lirticuectio-

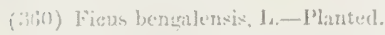

(ai1) Yicus retusa, b.- l'anten.

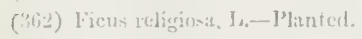

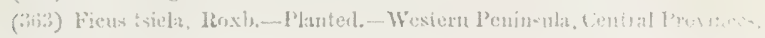
Cuslon.

7). Ceratopleyllacere,-

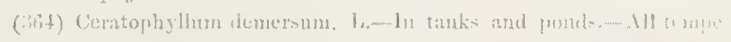
ant inpijcal regions.

\section{Hyirociurilucen.--}

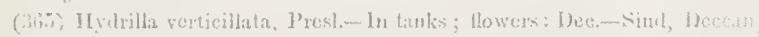

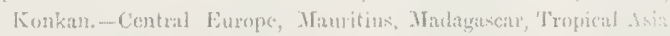
and Austualiat.

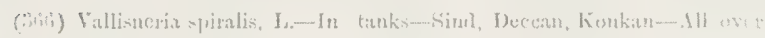

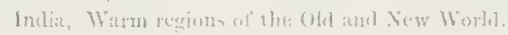

Litincer.-

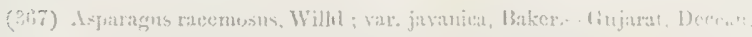

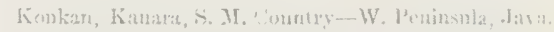

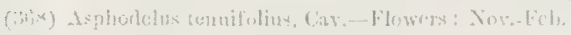

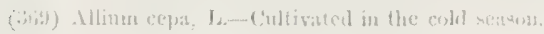

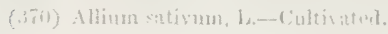

$\because 1$ 

74. Commelinztcere--

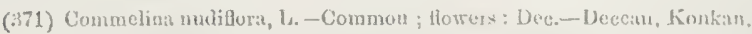
S. M. Country-In many tropical and subtropical countries.

(372) Commelina bengalensis. T.-Comminn; Howers: Sept.-Dec.

7ii. Pulmacer.-

(37:i) Phanix sylrestris, Roxb,-Cultivateal...'Throughout Indial.

(374) Cocus nuci ura, L.-Cultifated.

76. Ponlunacea:-

(375) Paudanus tectorius, Sulaud.-Plantud. Feal colst of the Imlian Peniusula, T3urma, Audarnans.

\section{Aroinlucers.-}

(376) Colocasia antiqnorum, Nichott. - Rare-Cultivateal in all hot countries.

78. T.emquacer

(377) Wolfin arrlizat, Wimn,--1n tanks, - Thuronghont India.

79. Nainatacers -

(378) Potanogreton indicus, lioxh,-In ponets and tarks.-Throughour tho.

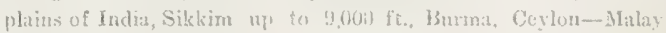
and Sandwich Istaneds.

(379) Naias minor, All.-Iu still swect waster,-'J'hroughont India and Ceylon-0)d thisld generally.

30. Cigpericeer.-

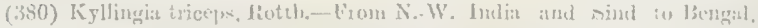
Burnat aud Ceslon.-Africa, China, Austrain.

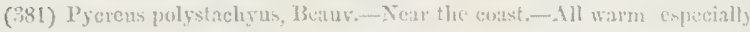
rataitime egrions.

(38.2) Cyperus harpan. I.-D'hroughuat India-- IIl warm regions.

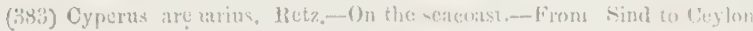
and Orisia, P'anjab-I'ersia, Aratria.

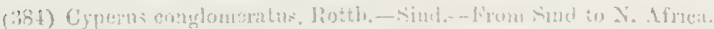

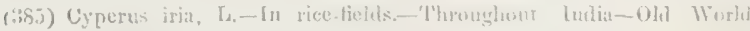
tyenentlly.

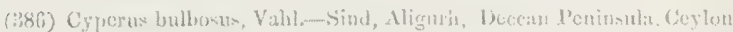
T'rengical Africal, Aria and Australia.

(387) Cyperus stoluniferus, lectz- -0 n the saudy sea shore-From sind th Ceylon, Malay Peniusula - Maturitius, Chiua, Malaya, Austualis.

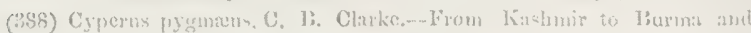
(eylon- 111 warm reçions exeept kinerpe.

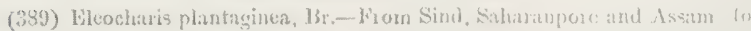
Ceylon and the Malay Peninsula-.'Lopies of the Ohd Work.

(390) Fimloristylis tetragona, 13r. - Throughout Indiz excent the North-

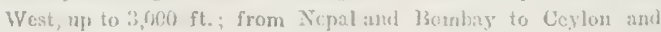
'Lavoy-L. Asia. Australia.

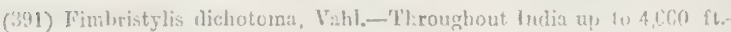
Warm recrions of the ()li Work. 



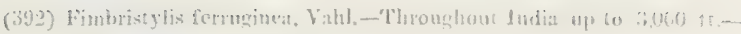
All warnoer resions.

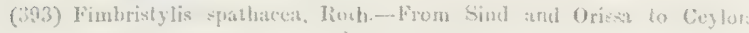
:mil Singuprere?

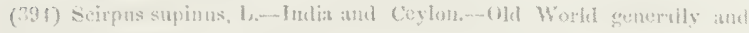
Americar.

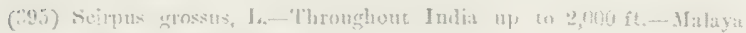
Tunkin, Philipuines.

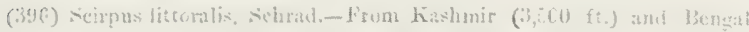
to Ceylun.-W. Isia, Ifricat, liurope, Anstralia.

91. Grotwizuere:-

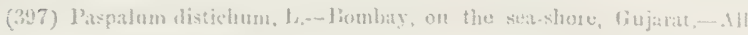
wer the troprics.

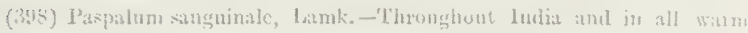
countrics.

(3ig) Panicum colonum, L. - Siud-All ofer India, Australia.

(t6o) I'anieum turgidum, Forsko-(injast, sind-Central India, l'blu chistan, Arabia. Higypt.

(\$01) Inuieum erus-galli, 1.. var. frumentaceun.-Cultivatcal.-Ifotim parte of Asia and 1 fritus.

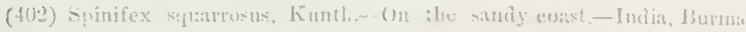
Ceylon, Javit, Chim.

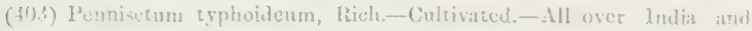
Ceylon.

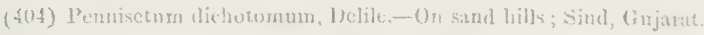

(toi) P'ennisetum setusum, ltich,-Gujast, Sind-Bundelkund, Contuii

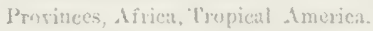

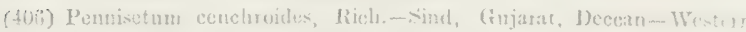
India, from Kashnir (1) the Cprer Cangetic l'lain, and somti ward, Tropical Ifriea, Canaies, sieily.

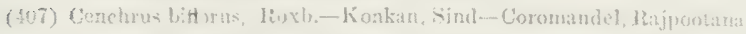

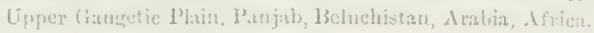

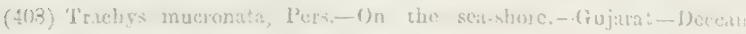
P'eninsula, Ceglon, Jattuin.

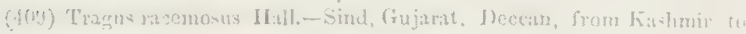

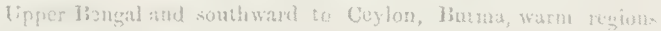
methertil!y.

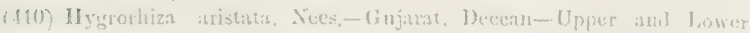

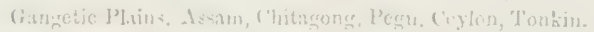

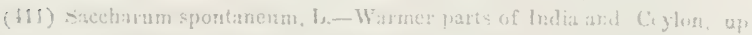

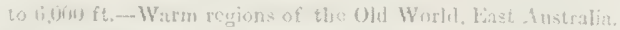

$(112)$ saccharum officinaram, J.-.-Cinltivated.

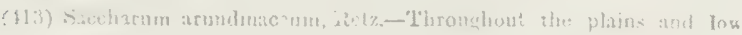
hellt of India-Ceylon, Chima. 

(f14) Laboum pilosum, Dalz. and (ribs, -Khandesh, i)cecan-Mysorc. Asirghar, Bellary, Chanda.

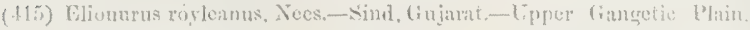

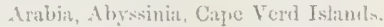

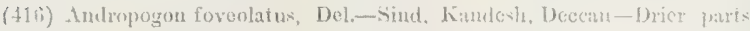

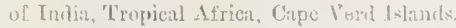

(417) Androporou sorghum, J3ot,-Cultisated - Warmer parts of Asia. Africu, Europe.

(415) Inilropogon ammlatus, Forsk,-Dectan, (iujarat-- Throughout the hills and viains of India, 'Tropicai Lfriea, China, Anstralia, Pacific.

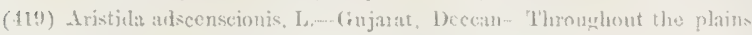

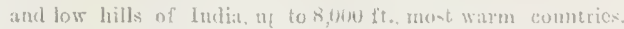

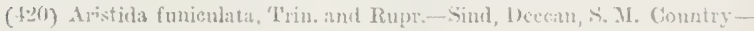
Plaius of the lanjab, hajputana, form the Konkan sout ward. Beluclistan, Aribiar. 'Tropical Africa.

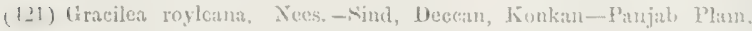
lizjputana, sind, Central India, Socotar, Nubia.

(12-2) Cynodon dactylon. Pers,-Thronghont Ludit. Burmat, and Ceylon. np to $5,(00) \mathrm{ft}$.- - All witrm comutries.

(1:,:) Chloris harbata, Swar.-Thronghout the platus of Inilia. Thuma, ind Ceylon-Tropies generally.

1.2f; Eleusine anyptiaca, Pers. - Theoughout the low ecountry of Indit. Buma, and Ceylon, Mladacen- Trarm Tegions of the Old Wondal.

(1 Li) Leptochlon chinensis, Viees,-Ciujatat, Kunkan-1'hroughout India and Barma, Ceylon, Malara. Cbina, Jitpan, Anstralia.

(126) J'hragnites karka, 'I'rin.-All over India, Ifghanistat, 'Tropical A-ia, Africa, Japrat Alustraliat.

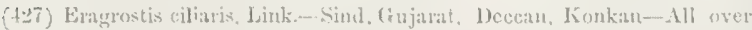
Inơil, Lrabia, 'T'ropical Africa.

(tis) Halopyrum inneronatum. Stapf,-Simd, Gujart, Konkan, KananCuasts of Western Ludia am Ceylon, Iribial. Tropical Africal.

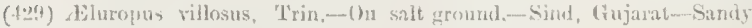
shores of the Decean P'eninsula and leyfor, salt plains of the ('anjab, Afghanistan, Persia, Arabia, Meclitomantan rogion.

(l:i) 'J'riticum vulgare, Is-Cultiruted.

(131) Hordeun rulgare, L., var. hexastichor, Aitchis-Cultimaterl.

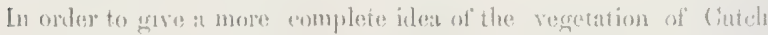

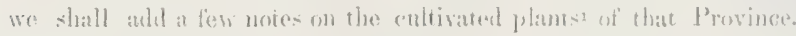

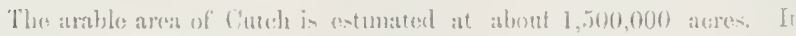

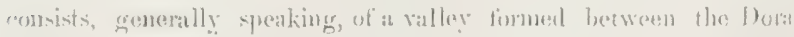

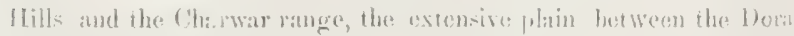

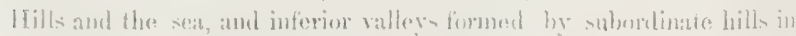





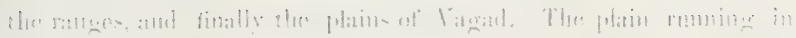

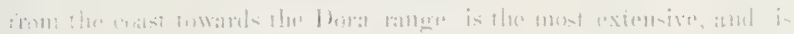

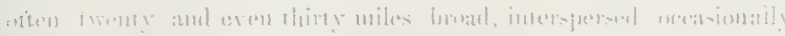

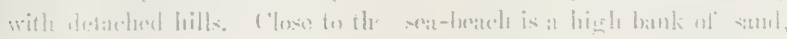

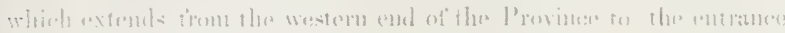

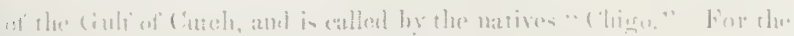

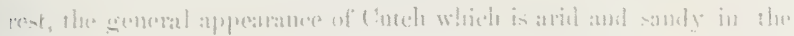

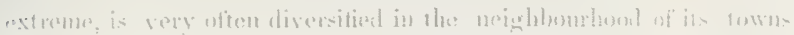

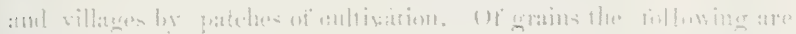

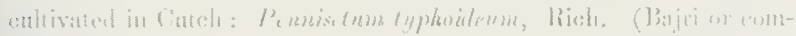

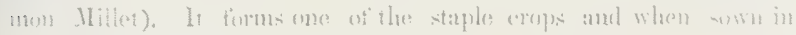

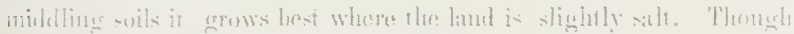

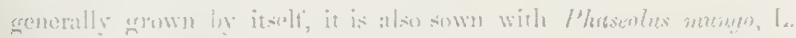

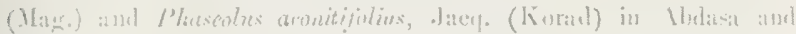

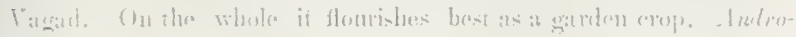

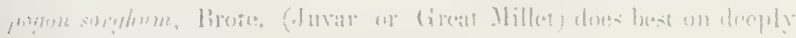

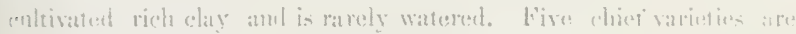

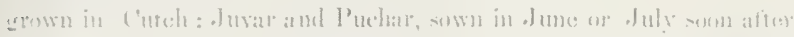

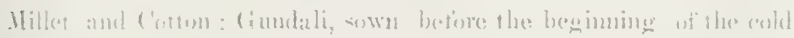

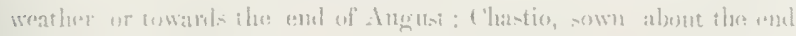

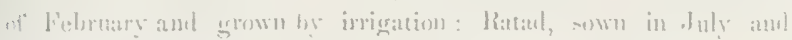

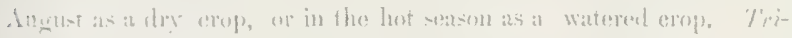

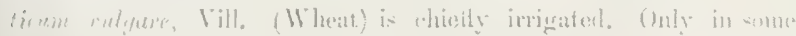

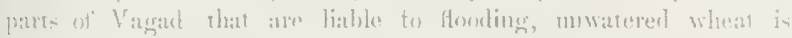

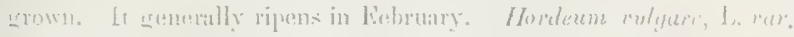

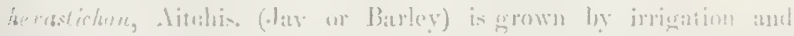

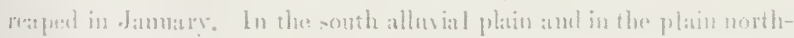

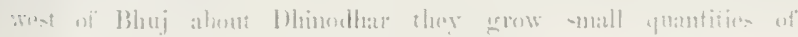

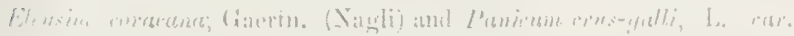

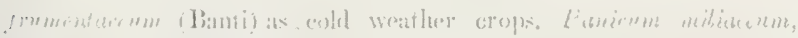

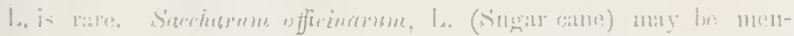

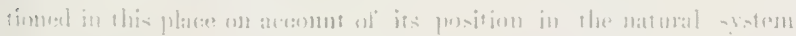

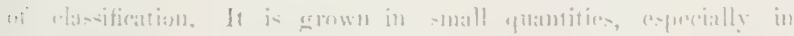

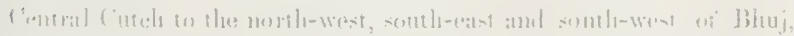

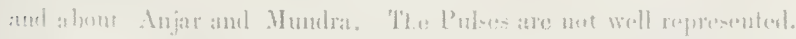

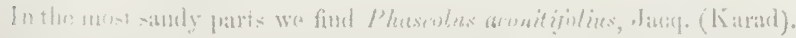

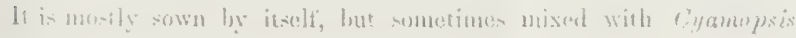

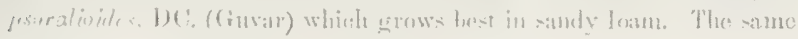



hind of soil is very suitable to Phascolus munoo, It. (Magr). Ciner

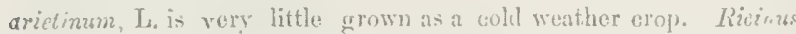
communis, L. is corimon all over the Provinee, but chiefly grown as an early crop in Varad. Kianthi, and anstern Abdasa. It is foten sown torgether witl Cotton. Scsamum indicum DC., and Gruizotia alyssinica, Cass, are grown in the rainy season, either by apelf, ats in Valgad and part- of Ablasa, or together with (boffon and Hillet in other places. Brassica cumpestris. In. rar. nupus (Rapeseed) is a cold weather crop, and grows aifles by itseclf or together with wheat. In eastorn Abdisat, Kanthi, and Vagad Gossupinm herhacen, Lo is exten-irely grown in elay soil. It is picked twice or thrice towads the end of liebruiry, in March or $\Lambda_{\text {pril. The common }}$ Indian vegetalles are cultivated in plenty, and the soil and climate

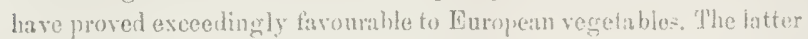
require only constant irrigation during the hot stasen to pireserve them in lusurance. The following are found in sorious parts of

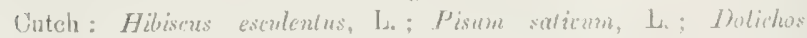

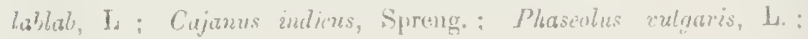
IToringa plerygosperma, Gacrtu.: Cucumis satinus, L.; Cucumis

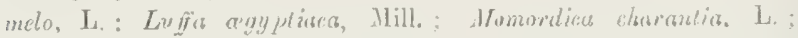

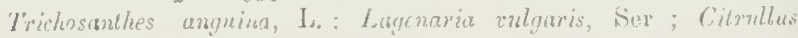
melgaras, Schrad.; Cicurbita moschata, Duchen ne: Cucurbita marima,

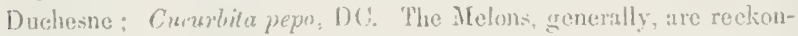
en superior to thase of any neighboming comatr. They grow in the dry beds of rivers when their rents the in a stream of water at fow inches bolow the surface. Their leaves and fruits being exposed to the double effect of at burning smen upon it scorching san l, the fout is hrought to the greatest perfeciom. The Melon is ripe in April, May and June. Other vegetables are: Marens carola, L. (Garrot); Apium :raveolens, I. (Celery); Solamm melongena, I.

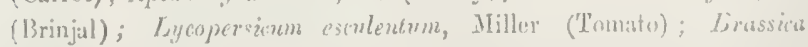
olerusta, L. with many varieties; Raphanas sulivus, I., Allimm eepa. $I_{3}$; Alliun sulimm, $I_{2}$. Besides, we find the following plints

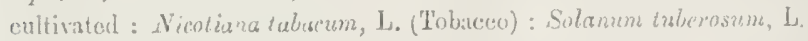

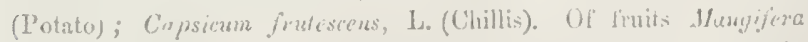

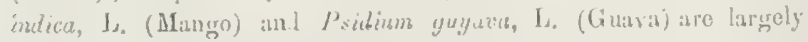
errown by the lielp of water. In some parts of the simly waterbearing yellowisht-lown soil wo find P/benix sylvestris, lioxb 



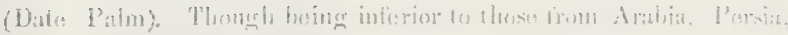

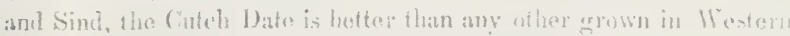

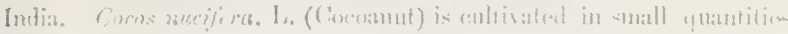

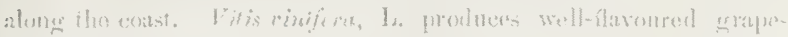

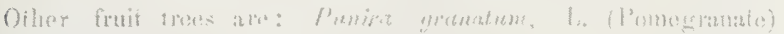

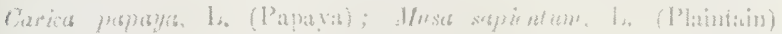

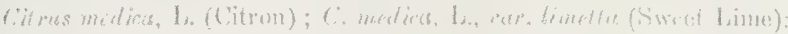

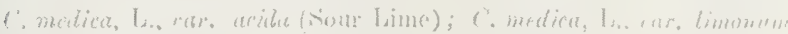

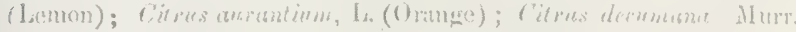

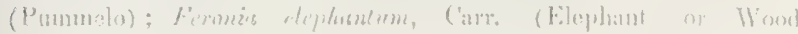

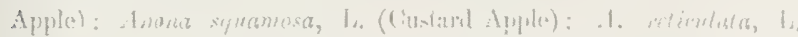
(Ramplaal).

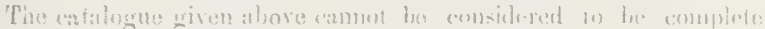

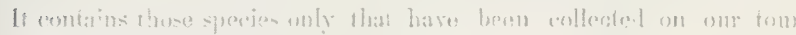

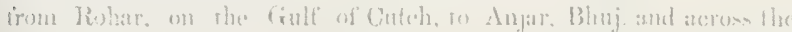

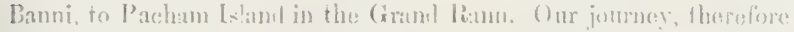

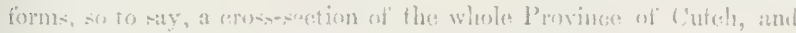

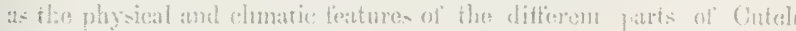

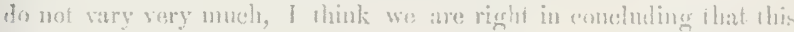

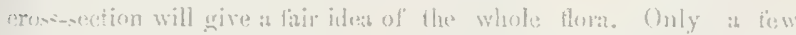

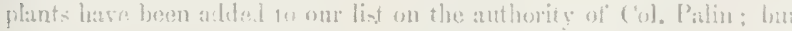
we have every reasoss on heliewe that they exelu in the simm regiun which we cammel ousolve.

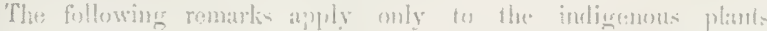

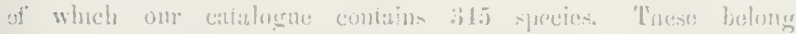

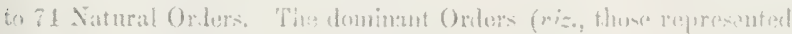

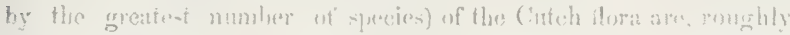

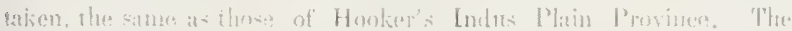

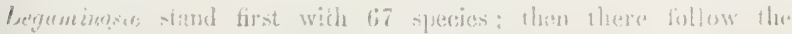

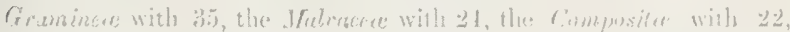

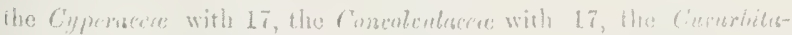

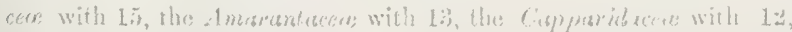

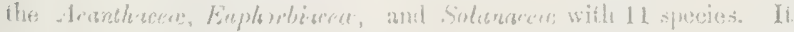

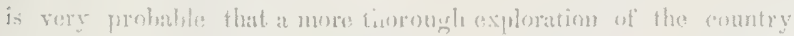

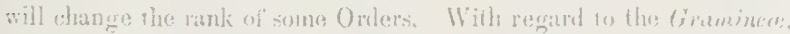

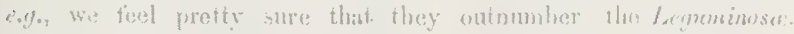

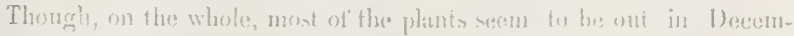

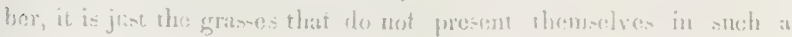



condition at that time as to turni-la suffeient material for their idenrifeation. Of the 345 indigenous plants alont 30 only are trees, 95. slumbs and andershmbs; all the rest are herbs, mostly perenuials. Almost all the plants have a while geographiend. distribution with regand to the Indian as well as to the extra-Indian region. No ente-

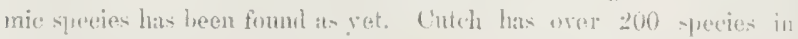

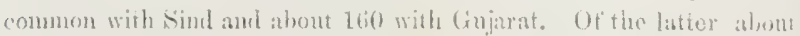
70 do not oeenr in Sind, whilst of the tomere oreer so latwe mot been

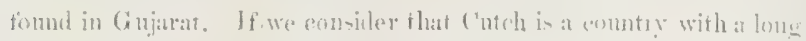
dry seasm, that the soil is motly sandy or rede, that salt is present in the wil not why along the seat shore and in the vicinity of the Great Ram, hut more or lewall over ile commery, we shall find it puite natural that most of the plante of (antehe cxhibit at remarkahle aneneral similuriy and agree sn having a lower rate of transpiratom than

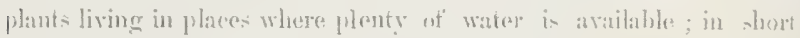

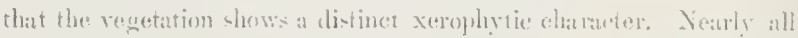
the structural featues hy which ramipiration is reatued mat he olserver in the flora of Gutcls: A thick euticlo men the apilemiof the leaves. reshetion of the number of semata, depmenton of the

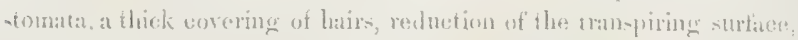

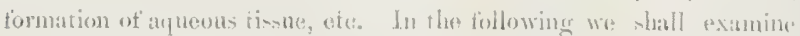
a number of plants in wheh the xerophytic eliarater in bent express-

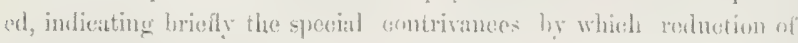
ran-piration is olotained.

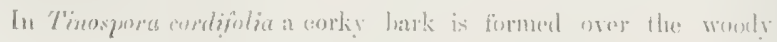

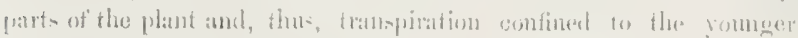
parts and the lowertas.

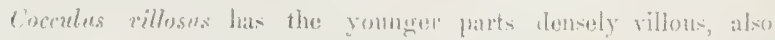

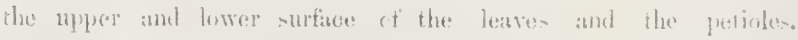

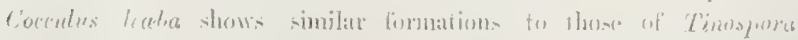

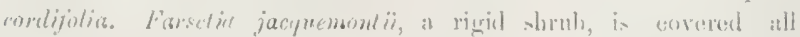

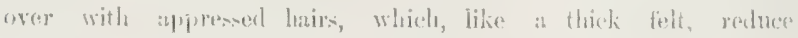

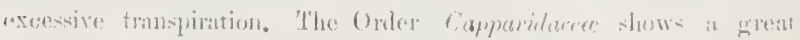

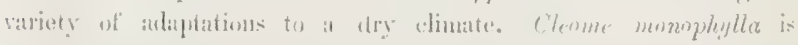

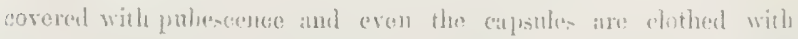
short stemt hairs. c'leome stochsione is partly protected hy haville

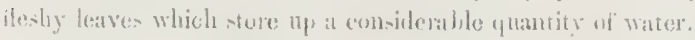

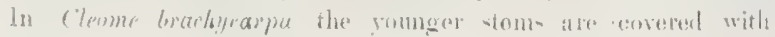





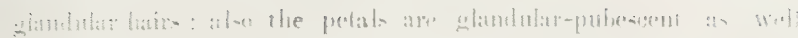

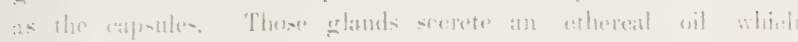

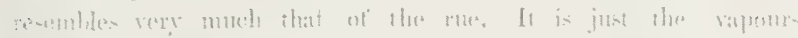

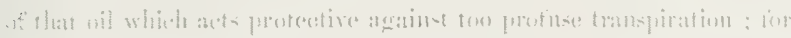

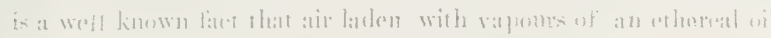

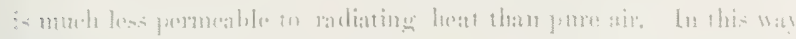

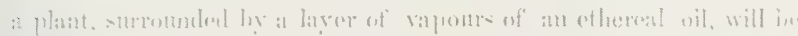

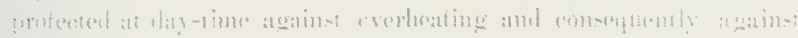

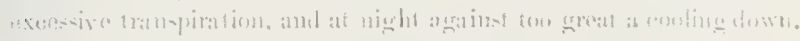

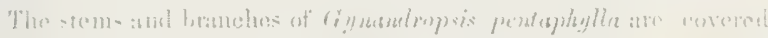

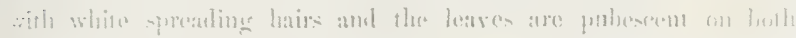

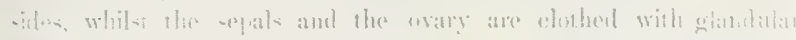
mair.

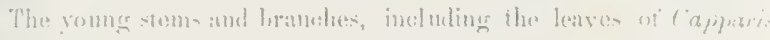

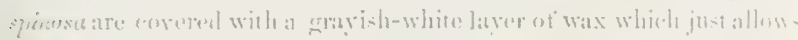

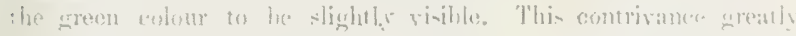

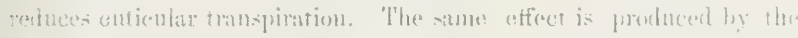

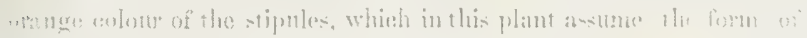

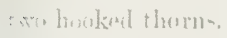

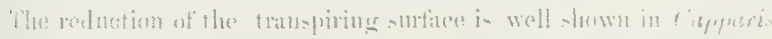

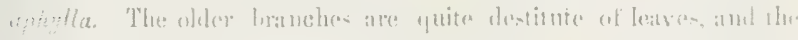

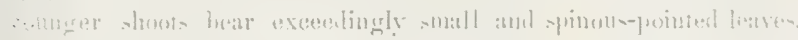

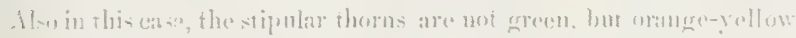

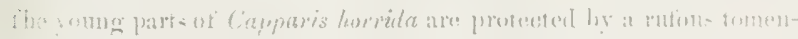

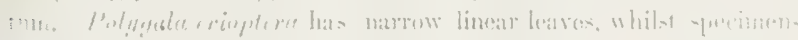

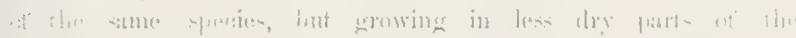

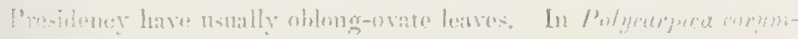

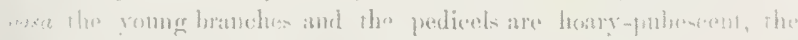

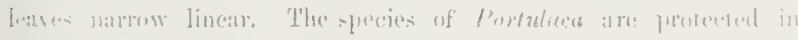

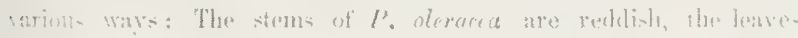

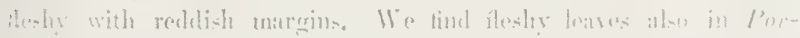

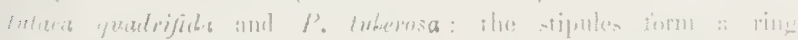

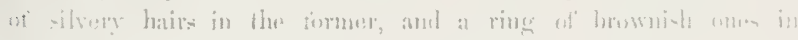
the list $1+1$.

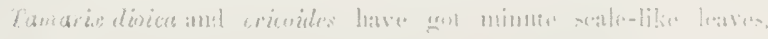

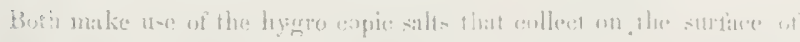

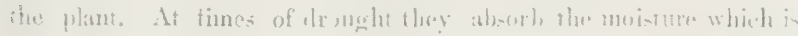

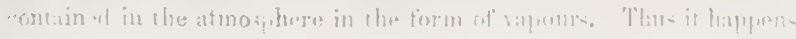

: 

that very often at night or in the early morning the green parts of the plant are covered with dew, whilst all the other plants in the neighbourheod are unite dry. The white (Just. that is reen at day-fine on the green branches and letres romsints ahicfly of chlorile of sodium and calcium ewhomate with some onher salime whitances.

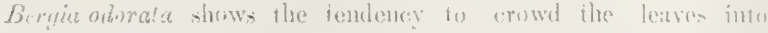
fivedeles. It is evident that by this mobns the leaver correl aud

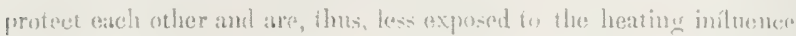

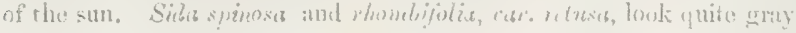

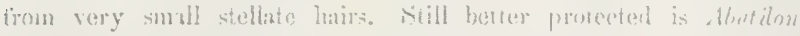
groweolens which, besites having long sponding hairs, is convered

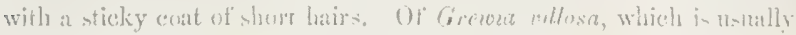

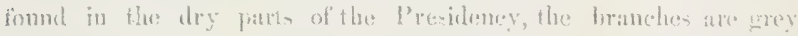

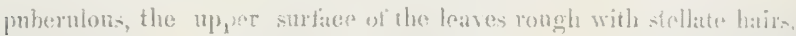
the lower velvety, the petioles villous and the stipules hairy, tho

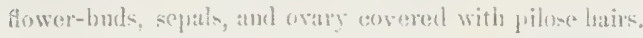

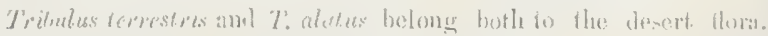
besides being protecterd by silky villous hair they perform some

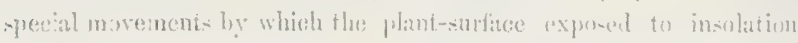

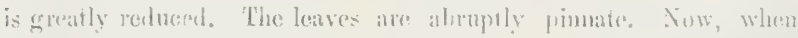
the beat is rising at nom or in the attemerom, the leateds begin to tum romd their own axis and, at the sume time, upwards rount the common rasehis, till they are lying in one vertical plain with these upper sides iomehiner atach oaher.

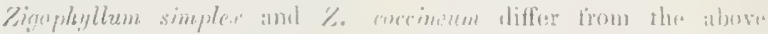

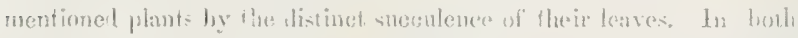

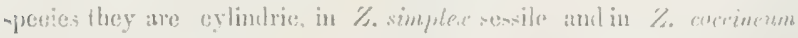

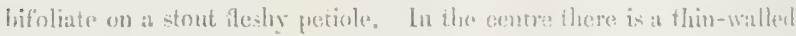

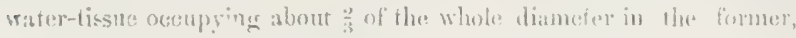
and about $\frac{1}{2}$ in the latier. At the same times, the somalta are de-

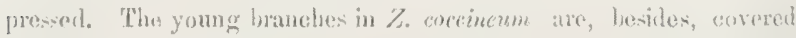

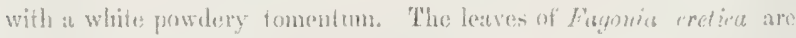
thick approaching the type of suesentent plimis. The onter walls of the prolygonal eells of the apidermis are very mush thickoned.

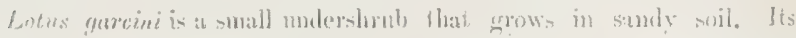
branclues are covered with grey silly latirs: the leatets are extrenely small and fleshy, both sides are riluthul with grey hatirs. Many 



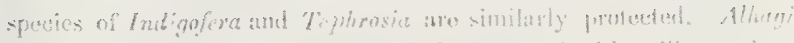

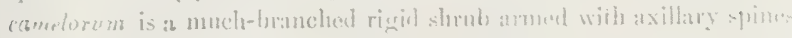

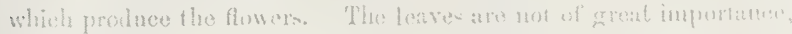

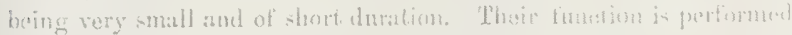

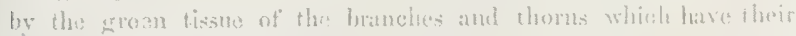

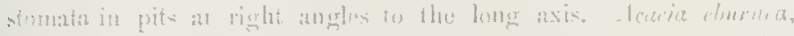
indigenous in the elry parts of India, has the youmg hamedies purplish-brown : the leaves atre very suatl and the longe ripular

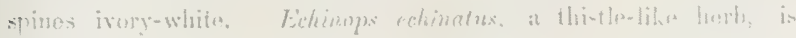

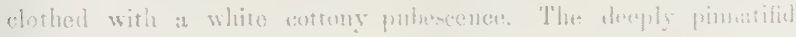

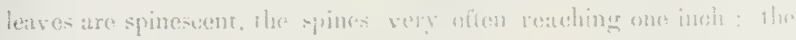
involueres are suremulerl by strong white bristes atnd the intar-

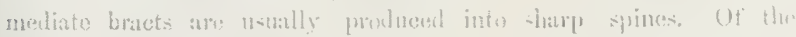

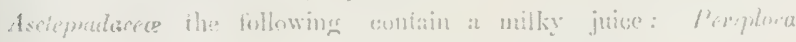

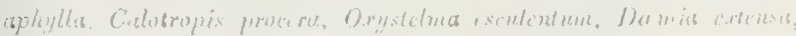

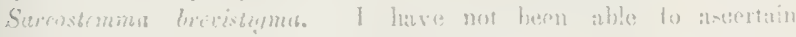

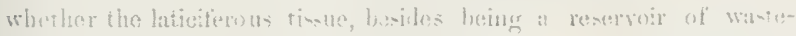
products, conkains ateo plastic substances 'Tlue litet that so miny

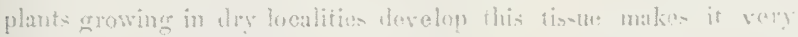

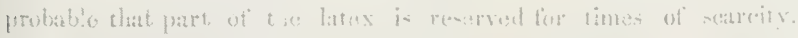

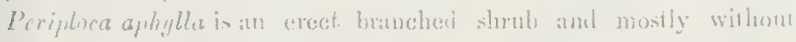
any leaves: hut when thes: are present they are extremely suall and thick. Calotropis procenterrows much higher in Guech than

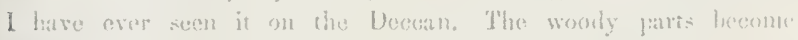
quite corky, whilst the young branches aud leaves are corered with

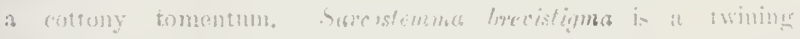
jointed shrub without leaves: tran-piration is confined we flue grean

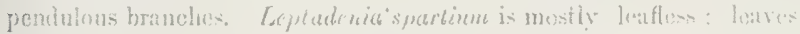
are seen sometimes on younes shorsts.

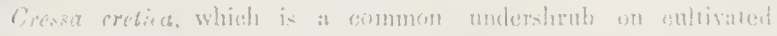
fields and blown sand, can be recognizest by the great. number of very small leaves flat are ooverest atl ower with a whiti-i arust of a hygroseupic salt. This is secreted in solutum ly grandulur hairs that are to bes found in defrensions on buth sidenof the leaves, It is not very probalile that the witer -njpliend by the ab-orling action of the sall is sulfictent in timen of

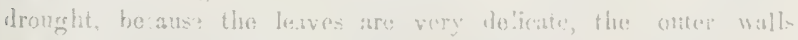
of the epidermal colls thin and the eromatit not depresesest at 



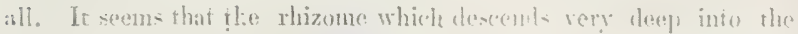
whistratum funishes the necessary moistur.

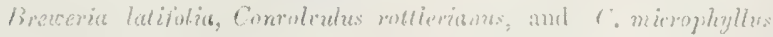
ane small undershubs confined to the dre parts of the Pres-

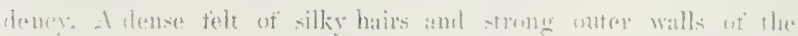

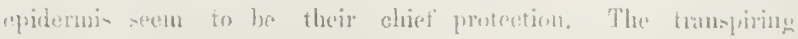

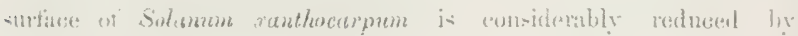

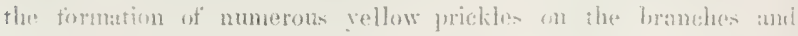

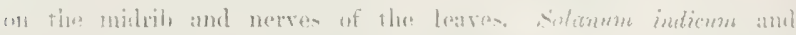

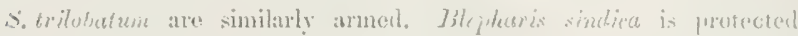
in rarions wass the stem is motly very short, often soaroly

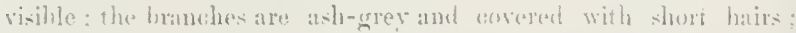
the leaves nes small linear and sessile, rough with siff hairs, and have the maghin bent batk : at the hase of the leaves there are n. metimes some small spinous teeth : the hrats are buiry on both -ides with at spiusus point, the upper part in armed with recument shape spime: the brateoles are hairy and ciliate: the outer and

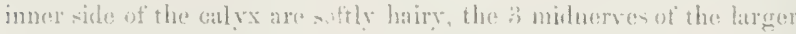

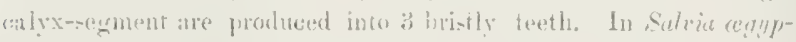

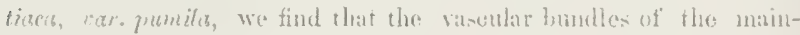

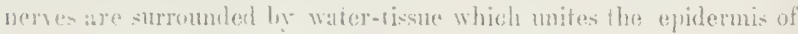
the uppere sich of the leatre with that of the lower one. As to

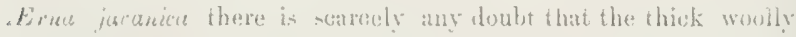
telt (o) vering almost all the part of the plant, is pretective in tinction. In Siblicomid brathible, a jointed hanched shrub, transpiration is entirely confined to the sreen branches, there being only

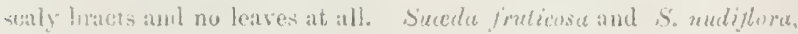
which matally gros in saliue places, hat feshy leaves. A transwerse antion shows that the greatest part is oecupaed by water-lisste.

Bofure concluding this paper I should like to make a fow remarkis un the flom of the firand Bam, as it has heen suggested that at more acentute linowledge of the regretation of that part of the conutry might heip to decise the question as to the origin of the kanm. I samnot give a botter description of that interestiug

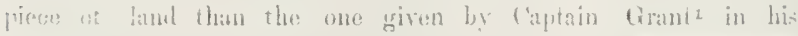

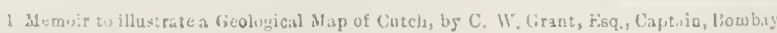

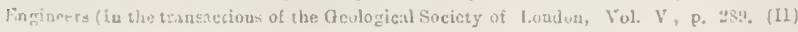
-erigus? 



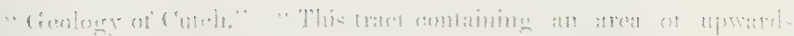

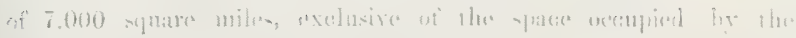

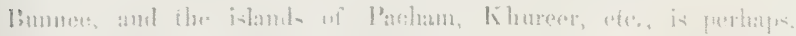

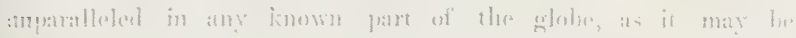

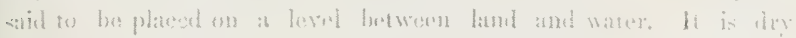

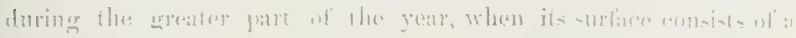

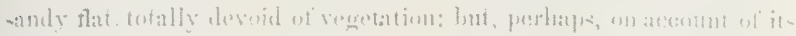

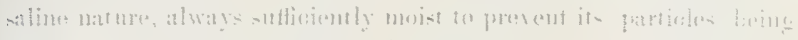

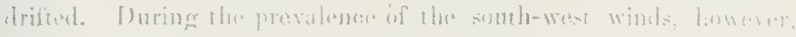

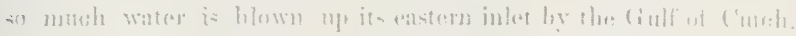

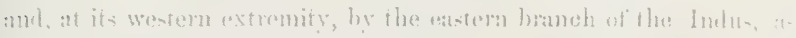

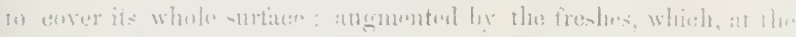

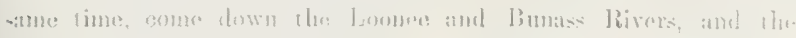

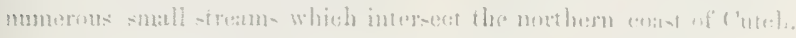

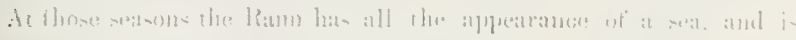

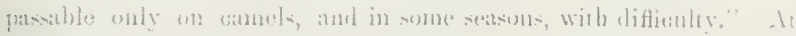

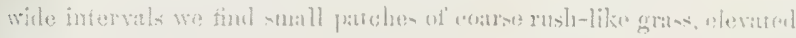

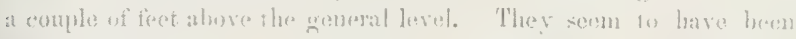

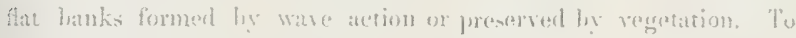

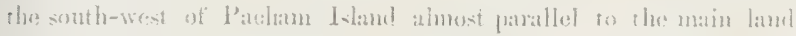

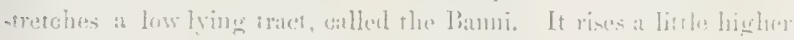
than the remainker of the liann and is about tis miles longe and fioms

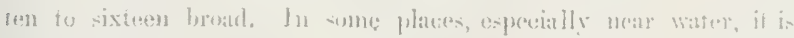

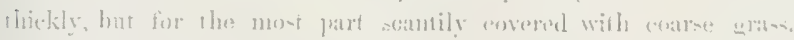

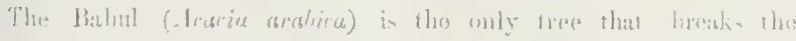

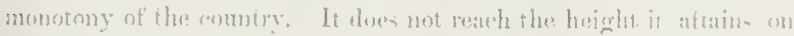

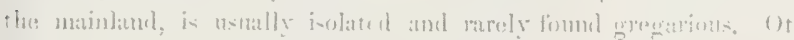

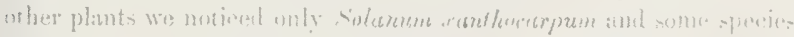

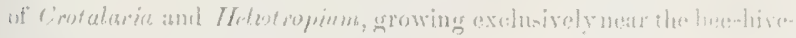

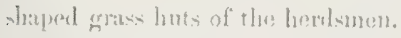

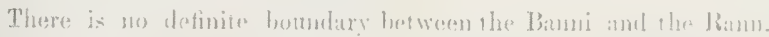

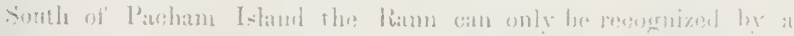

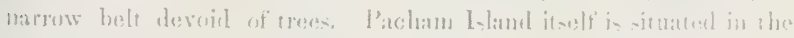

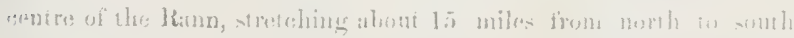

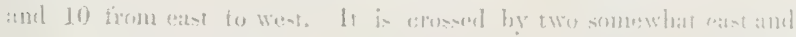

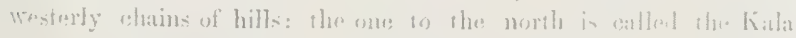

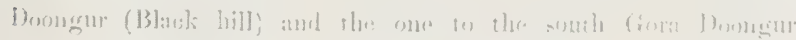

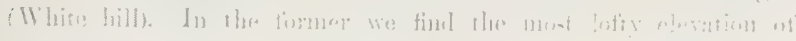



Cuich, the Pacham P'ir which rises directly from the Ranu to as height of 1.137 feet. A most extensive riew can be olituined from the summit of this mountain. Boyond a waste of salt and water the Parkur hills are visille, and to the south and south-east appear the dark surfuce of the bami and the (nteh hills. 'The istand is surrounded by at margin of low grount of no great. width, but narrowest where the mountains rise most steeply from the Ram on the northprin side. Shoets of hemefitis laterite overlap and wrap round the juassics on the west and south of the low grouml, sometimes atsociated with atrtly rouss and beds remoubling voloanie ash. Figher up the berls consist of fime white and light colonred silicious. sandatone with calcalreun bands and sabdy slightly ferruginous purple heds: prile flagery sindstmes also noenr.

This is unt the plate to enter intos at discussion ats regatrds the

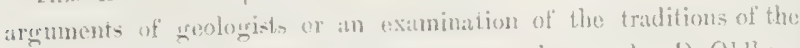

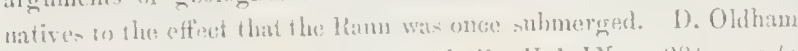

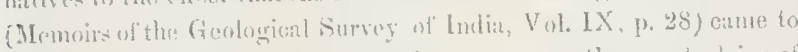
the forlowing conclusions: "Tho whatever catuses the great pltans of

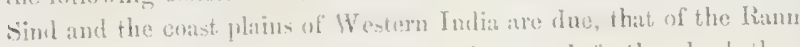
may aloo he ascribed. It origin must be tracest further back than

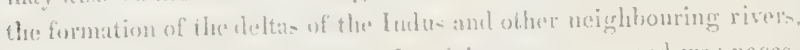
hecutues something in the nuture of a plain or open ground was neces-

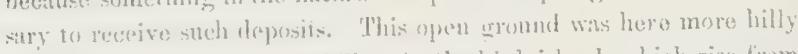
than to the north in all probubility, tar the high islants which rise from the Rimn are evidenily lut the modified summits of an older surfuce; and the silfing-mp of the sam-intet which it formed was only the

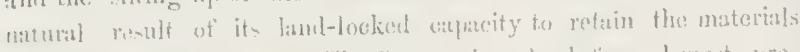

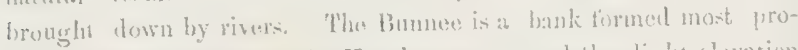
hably by the discharge of the Kutch stream.; ; and the slight elevartion en messe which subjected the olnt shore-thposits to denudation has vided the tenderey of the hasin to retain tramsported saliment, which must accumulate yearly under present circumatumes until the river:

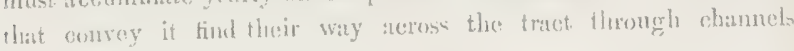
Irtwersing an alluvist plain."

Su fir treology on the origin ol the Bann. Are wo able from the sandition ant characters of the present flor. of Pabcham Is'and to

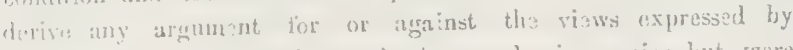

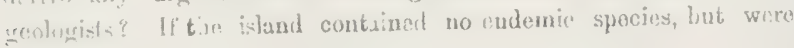





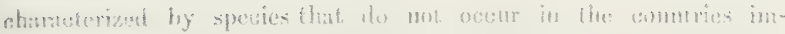

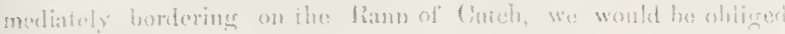

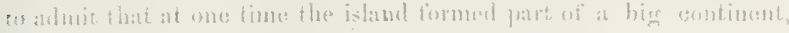
that, afterwarls, its immediate smrometings weres submergal, ambl that what is now Sind, liajputana, Guzerat, and Guteh, was changend

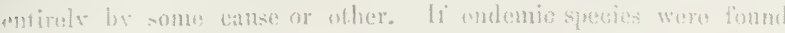

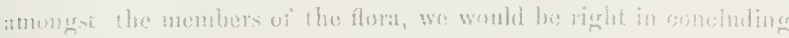

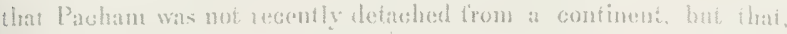

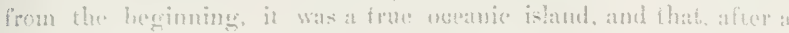
long interval, the land began io riar and to form round lactuan Iste. Soither of the lwo conditions is fultilled with regsm to the

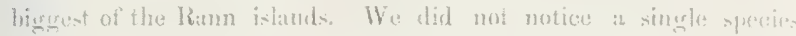

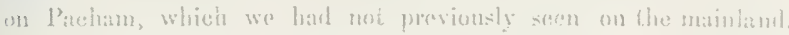

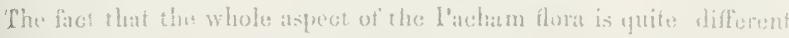
from that at any oflex part of Cunch examined by ns is not due to

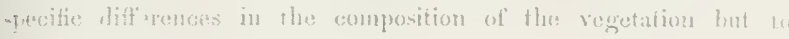
differences in the combination and develument of its members, in

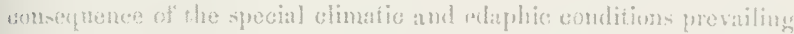

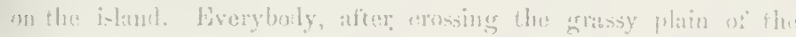

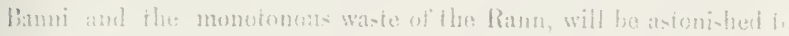

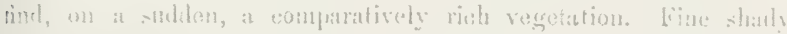

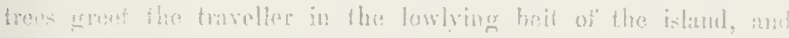

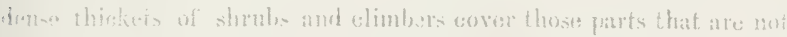

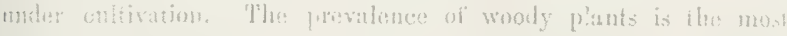

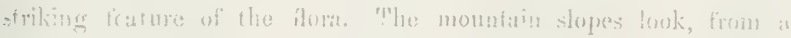

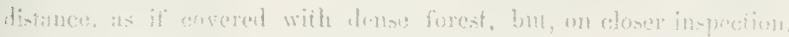

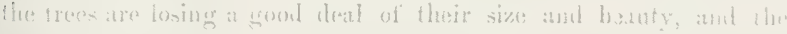

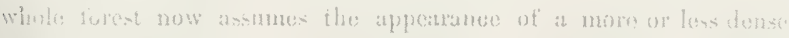

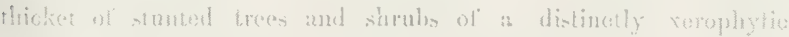
chanacter. The harbaceons regetation is very seatuly: only in arevices, where some soil has iccumulated, nature hats sneceded in

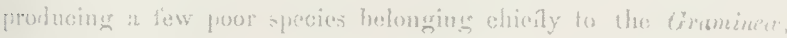

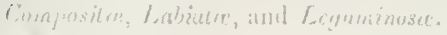

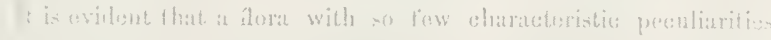

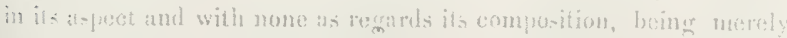

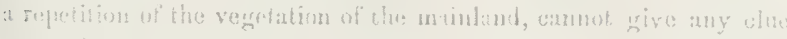

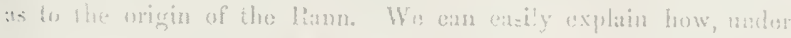
endinions similar to those of thes present. dity, the whole florat of 

17i JOERNAL, BOMBAY NATURAL HISTORY SOCIETY, DOI. XIX.

Pasham Yaland may have dercloped in the compe of at tew centuries: we have only to remember the difterent methods that are enmloyed by plants for the purpose of seed-rlispersal. If this be once athitted it is difficult to see how we shall measure goological times hy the moments of a flurat that may posibly be of yectertay. 



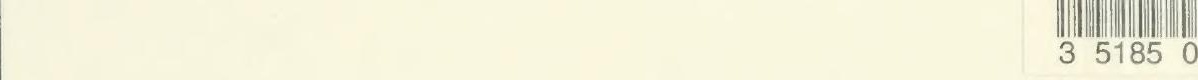


